www. revist a d y o.com

\title{
Actualización del universo de marcos de trabajo de arquitectura empresarial. Identificación, caracterización y evaluación
}

\author{
Maykel Manuel Chávez Rodríguez, Leisis Villar Ledo \\ https://doi.org/10.37610/dyo.v0i72.584
}

Recibido: 10 de Diciembre de 2019

Aceptado: 22 de Julio de 2020

\section{Resumen}

La arquitectura empresarial se ha desarrollado en los últimos años en gran medida por el desarrollo de marcos de trabajo que contribuyen a su aplicación y estandarización. En el presente trabajo se investigó sobre el universo actual de los marcos de trabajo de arquitectura empresarial mediante su identificación, caracterización básica y evaluación. La identificación se realizó mediante el análisis de una amplia base bibliográfica y tuvo como resultado un listado final de 160 marcos de trabajo. La caracterización básica se realizó para todos los marcos identificados cubriendo los aspectos esenciales como es el año de publicación, el sector de desarrollo y el autor o propietario. Finalmente se evalúan los marcos de trabajo identificados a través de un grupo de criterios establecidos por un método específico desarrollado por el autor. En la investigación se logró abordar la variedad y diversidad actual de los marcos de trabajo de arquitectura empresarial, aportando una visión integral y abarcadora del objeto de estudio. La base de información y documentación resultante de la investigación, así como el método de evaluación propuesto, son útiles para todos los usuarios que se acercan a la arquitectura empresarial con el objetivo a encontrar respuestas a sus necesidades de mejora e iniciativas organizacionales.

\section{Palabras clave}

Empresa, arquitectura; arquitectura empresarial; marco de trabajo.

\section{Introducción}

El factor tecnológico ha dejado de ser un privilegio de unas pocas organizaciones de clase mundial para convertirse en un requisito básico en cualquier industria (Minoli 2008), dando paso a un mundo digital (Jayakrishnan, Mohamad, y Abdullah 2019). Las organizaciones han aumentado las capacidades para alinear las tecnologías de la información con las estrategias comerciales (Kotusev 2017). Esto ha sido posible en gran medida por la introducción de la arquitectura empresarial, que ha funcionado como una solución para manejar la complejidad de empresas grandes y pequeñas en todo el mundo (Sessions 2011).

\footnotetext{
凶 Maykel Manuel Chávez Rodríguez * maykel.chavez@etecsa.cu

Leisis Villar Ledo **

leisis@int.cujae.edu.cu

iD https://orcid.org/0000-0001-5842-4111

* Empresa de Telecomunicaciones de Cuba S.A. https:// www.linkedin.com/in/maykel-manuel-chavez-rodriguez7a80a741/ https://www.researchgate.net/profile/Maykel_Manuel_Rodriguez

** Universidad Tecnológica de la Habana "José Antonio Echeverría" (CUJAE).
}

Han pasado más de 30 años desde el famoso artículo de J.A. Zachman (Simon, Fischbach, y Schoder 2013) publicado en la Revista de Sistemas de IBM en 1987 (Zachman, 1987) pero las potencialidades de la arquitectura empresarial no han parado de generar interés (McDowall 2019, Sessions y deVadoss 2014). Este es para muchos el momento en que surge la arquitectura empresarial (AlDhaheri 2011, Sessions y deVadoss 2014, Kotusev, 2016, Roach 2011) y para otros es el punto donde se forma su núcleo inicial que al pasar de los años evolucionaría desde los sistemas de información (Orabona 2016, Farazmand y Moeini 2011, Marini 2019, Cretu 2014). En lo que sí existe consenso es en que ha evolucionado enormemente como concepto y como disciplina de negocios (Marini 2019, Simón et al., 2013), convirtiéndose en la actualidad en elemento fundamental en los programas de mejoramiento de diversos sectores e industrias. Su repercusión se ha notado en la implementación de la estrategia empresarial (Jayakrishnan et al. 2019), en la transformación digital (Laschitza y Undén 2017) y en la creación de procesos ágiles (Lauvrak, Michaelsen, y Olsen, 2017).

Comúnmente junto a la arquitectura empresarial se encuentran modelos que funcionan como marcos de trabajo para representarla y formalizarla. Estos se han desarrollado casi a la par de la disciplina (Bui 2017). Desde que John A. Zachman introduce por primera vez la visión de lo que debe ser un marco de trabajo de arquitectura empresarial (Zachman, 1987), estos han evolucionado trayendo muchos 
beneficios y aportes a las organizaciones (Schekkerman, 2006, AlDhaheri 2011). Los marcos de trabajo son abundantes en todas las ramas de la industria, ya lo había acotado Schekkerman en el 2004 cuando utilizó el término “jungla” para referirse metafóricamente al ámbito donde estos han estado surgiendo (Schekkerman, 2004). Esta "Jungla" hoy en día continúa creciendo. La variedad de los marcos de trabajo de arquitectura empresarial es uno de los principales inconvenientes para su aplicación práctica. Suelen encontrarse en todos los sectores de actividad, en todas regiones del mundo y en todo tipo de industrias, lo que se convierte en un verdadero "dolor de cabeza" para los especialistas o directivos que tratan de utilizarlos.

La presente investigación tiene como objetivo caracterizar el escenario actual de los marcos de trabajo de arquitectura empresarial en cuanto a la variedad, disponibilidad y métodos de evaluación. Los enfoques de estudios similares consultados en la literatura presentan tendencia hacia la visibilidad de un grupo muy selecto de marcos de trabajo. Estos enfoques no representan la complejidad real y limitan las opciones que tienen arquitectos empresariales o profesionales interesados para seleccionar la variante más adecuada a sus necesidades prácticas.

El trabajo está constituido por tres secciones: sustento teórico de la investigación, métodos de la investigación y resultados.

Sustento teórico: Debido a la existencia de gran variedad de definiciones del objeto de estudio se analizan las dimensiones conceptuales que según al autor definen una línea teórica válida para la investigación. Se analizan conceptos de arquitectura empresarial, marcos de trabajo y se hace una revisión de los métodos para la evaluación de los mismos.

Métodos de la investigación: Se establecen los métodos, técnicas y herramientas utilizadas durante la investigación.

Resultados y discusión: Se presentan los resultados obtenidos. Primeramente se identifican y se caracterizan básicamente los marcos de trabajo de arquitectura empresarial. El análisis general es en base al país de origen, fecha de surgimiento y sector de procedencia. La evaluación de los marcos se realiza mediante un método propuesto por el autor y los resultados de la evaluación se presentan y analizan de acuerdo a los puntos fuertes y débiles de los marcos evaluados.

Todos aquellos usuarios de la arquitectura empresarial, tanto directivos como profesionales de diversas ramas, tienen en el resultado de este trabajo información útil para orientar sus decisiones en materia de utilización y explotación de los marcos de trabajo. El universo de marcos es expuesto de forma integral, actualizada y documentalmente verificada. Además puede ser útil tanto para el sustento de la elección de uno o varios marcos de trabajo como para explotar las posibilidades de cada uno teniendo en cuenta las necesidades de cada organización. Esta investigación constituye una guía completa para observar integralmente el complejo mundo de los marcos de trabajo y explorarlo más profundamente en busca de soluciones o iniciativas basadas en la arquitectura empresarial.

\section{Sustento teórico de la investigación}

\subsection{La arquitectura empresarial. Conceptos, beneficios y utilidad}

Académicos y organizaciones han dado multitud de interpretaciones de la expresión de arquitectura empresarial (Ajer 2020, Kotusev, Singh, y Storey 2015, Abdallah y Abran 2019, Nilsen 2012, Saint-Louis y Lapalme 2018, Marini 2019b, Ferreira de Santana 2017, Pähler, Morency, y Lapalme 2019, Rogier, van de Wetering, y Joosten 2020). Hasta el momento no existe una definición universalmente aceptada (Dang y Pekkola 2017, Ross, Weill, y Robertson 2006, Niemann 2005, Winter y Fischer 2007, Perroud y Inversini 2013, Sidorova y Kappelman 2010, Nygård y Olsen 2016, Kotusev et al. 2015) a pesar de que en muchos de los conceptos existe cierta superposición (Vermeulen 2013). Según (Saint-Louis y Lapalme 2018) esto indica que la arquitectura empresarial es una práctica inmadura. Pero más allá de la ambigüedad del concepto, esta se ha destacado por facilitar la integración eficaz de la estrategia, personal, negocios, datos y tecnologías de la información (Moscoso, Paredes, y Luján 2019, Abunadi 2019, Niemi 2006, Laschitza y Undén 2017, Llanos y Ortiz 2011).

La definición de arquitectura empresarial surge de una analogía con la construcción (Ferreira de Santana 2017, Vermeulen 2013, Perroud y Inversini 2013), ya que el término arquitectura se refiere al diseño de cualquier tipo de estructura (Alwadain et al. 2014). También utilizada en la industria del software (Vermeulen 2013), el término arquitectura llevado al ámbito empresarial evolucionó para describir el diseño o plano de una organización en sí misma (Marini 2019). Inicialmente se crearon con la noción de arquitectura de sistemas de información (Marini 2019, Orabona 2016, Kotusev, 2016), pero hoy su alcance es mucho más amplio (Simón et al., 2013) ya que son capaces de describir una arquitectura de referencia o habilitar la descripción de la misma (Tome y Costa 2010). En la amplia gama de definiciones de arquitectura empresarial resaltan aspectos comunes (Vermeulen 2013) como su enfoque holístico, la relación del negocio con las tecnologías de la información y el establecimiento de un plan detallado de migración de un estado actual a un estado futuro.

Enfoque holístico: La arquitectura empresarial puede considerarse un enfoque holístico (Hylving y Bygstad 2018, Werewka 2018, Nygård y Olsen 2016, Armour y Kaisler 
2017, Rogier et al. 2020, Qurratuaini 2017) que contiene una representación de toda la empresa (Fratila 2020, Aier, Fischer, y Winter 2011, Gampfer 2019, Winter et al. 2010, Fischer, Winter, y Aier 2010, Ajer 2020, Lê 2020). Este enfoque es la característica más importante de la arquitectura empresarial (Lankhorst 2009) ya que logra una comprensión integral de la organización que apoya el desarrollo exitoso de la estrategia (Kar y Thakurta 2018, Hylving y Bygstad 2018, Erdenebold, Rho, y Hwang 2019) y el manejo de la complejidad (Niemi 2016, Sessions 2008, Niemann 2005). La arquitectura empresarial se considera como un conjunto de descripciones lógicamente vinculadas y estructuradas (Zachman 2011), que se basan en subdominios que entregan diferentes agregados (Aier et al. 2011, Winter et al. 2010, Fischer et al. 2010).

Relación negocio $\mathbf{y}$ tecnologías: La arquitectura empresarial es la lógica de organización (Ross, Weill, y Robertson 2014, Kamoun 2013) de los procesos de negocio e infraestructura de tecnologías de la información (Lankhorst 2009, Abunadi 2019, Niemi y Pekkola, 2013, Aier y Schelp 2010, Bernard 2012, Cabrera et al. 2016, Sukur 2020). Esta refleja la armonía (Azizi y Sumitra 2019), la integración (Randall 2018, Priyanto 2013) y la estandarización de los requisitos del modelo operativo de la organización (Ross et al. 2014, Minoli 2008). Actúa como un eslabón fundamental para la alineación del negocio con la tecnología (Niemi y Pekkola, 2013, Okhrimenko 2017, Niemi 2016, Tamm et al. 2011, Banaeianjahromi y Smolander 2017), considerándose como un instrumento importante para la efectividad empresarial (Lankhorst 2009). La arquitectura empresarial considera el uso eficiente que se da a la tecnología (Sari, Rahmawati, y Harafani 2019, Caruso 2019, Lukhele, Ngassam, y Osunmakinde 2015, Armour y Kaisler 2017) en los procesos comerciales (Sultanow et al. 2016) y orienta objetivamente las inversiones tecnológicas (Banaeianjahromi 2018, Proper et al. 2012, Sari et al. 2019). También es capaz de aportar flexibilidad (Laschitza y Undén 2017), interoperabilidad (Pasaribu, Sipahutar, y Situmorang 2019), eficiencia y sostenibilidad (Aier y Schelp 2010).

Plan detallado de migración: La arquitectura empresarial se define como un plan maestro (Perroud y Inversini 2013, Schekkerman 2005, Open Group 2009) que contiene una descripción del estado actual de una organización (Kotusev 2019), un plan de transición y la descripción de un estado futuro (Lange, Mendling, y Recker 2012, Open Group 2011, Alwadain et al. 2014, Simon et al., 2013, Tamm et al. 2011, Bernard 2012). Apoya a las organizaciones en un entorno cambiante (Kotusev 2019) al definir una base de pensamiento (Sofyana y Putera 2019) para la toma de decisiones y el desarrollo de la estrategia (Erdenebold et al. 2019, Chakraborty y Sarkar 2017). La arquitectura empresarial incluye una arquitectura base, una arquitectura destino y un plan de secuencia (Stroud 2019). Actúa modelando elementos de las organizaciones (Alwadain et al. 2014), desarrollando capacidades de la empresa y modulando su comportamiento (Giachetti 2015).

Existen muchos aportes de la arquitectura empresarial que la convierten en una disciplina de negocios imprescindible. La capacidad de definición de un enfoque para gestionar la complejidad (Sessions 2008), la utilidad para comprender de forma integral a la empresa (Lauvrak, et al. 2017) y su visión integrada de los procesos de negocio (Niemi y Pekkola 2019), son algunos de estos aportes. La arquitectura empresarial crea infraestructuras (Baharudin Jusuf y Kurnia 2017), aporta agilidad en la implementación de estrategias (Londoño 2014), aporta una mejor comprensión de la organización (Bernard, 2012) y proporciona soporte en el contexto normativo (Proper et al. 2012). Además aumenta la capacidad de integración de la tecnología con el negocio (Theuerkorn 2005, Chorafas 2002); Abdullah, 2017), facilita el logro de los objetivos empresariales (Foorthuis 2012), entre otros muchos beneficios.

A pesar del impacto que está teniendo, la arquitectura empresarial es considerada como un campo en evolución (Sessions y deVadoss 2014) en un entorno donde los costos para desarrollar sistemas de información son cada vez más bajos (McDowall 2019). Las mayores críticas recibidas por la arquitectura empresarial se relacionan con las pocas evidencias de su impacto real en las organizaciones. A pesar de la popularidad en la última década, no es fácil encontrar ejemplos de arquitecturas desarrolladas con éxito en una organización (Banaeianjahromi y Smolander 2017). La mayoría de las organizaciones durante la implementación de la arquitectura empresarial se enfrentan a problemas como la adopción de enfoques dispersos, la utilización de arquitecturas obsoletas (Rouhani y Nikpay 2012), la falta de uniformidad de las iniciativas (Saint-Louis y Lapalme 2018) y la falta de flexibilidad (Hylving y Bygstad 2018). Existe poca investigación sobre mecanismos para clasificar, dimensionar, comparar u organizar el análisis de arquitecturas (Oliveira Barbosa et al. 2019). Pero el futuro de esta disciplina no es para nada incierto. La amplia diversidad de herramientas de visualización actuales (Rehring y Ahlemann 2020), la transformación digital e incremento de la agilidad (Hylving y Bygstad 2018) y la necesidad de responder a las capacidades emergentes de los dominios comerciales (Alwadain et al. 2014) hacen de la arquitectura empresarial una disciplina necesaria e imprescindible en el futuro. 


\subsection{Marcos de trabajo. Conceptualización e implicaciones prácticas}

Existe consenso sobre la importancia que han tenido los marcos de trabajo en el desarrollo de la arquitectura empresarial (Vargas 2015, Thea Nisaa', Asti, y Muharman 2019, The Open Group 2018) y muchas organizaciones, tanto privadas como gubernamentales han incursionado en esos para su uso operativo (Nilsen 2012).

La proliferación (Carr 2018) y diversificación de modelos arquitectónicos ha llevado a un conjunto amplio (Kimpimäki 2014, Qurratuaini, 2017, Minoli 2008, Okhrimenko 2017, Yoganingrum, Sensuse, y Murni 2013, Roach 2011, Vargas 2015, Halawi, McCarthy, y Farah 2018) y divergente de interpretaciones del concepto de marco de trabajo de arquitectura empresarial (Sanchez y Collado 2017, Stroud 2019). Estas interpretaciones varían, considerando a los marcos de trabajo como arquitecturas empresariales en sí mismos, meta modelos de arquitecturas o modelos donde se expresan y conceptualizan arquitecturas. Un marco de trabajo puede considerarse como una estructura lógica (Barekat, Nejad, y Alavi 2013) para categorizar y organizar múltiples artefactos (Mokone, Eyitayo, y Masizana 2019) que engloban un conjunto de asunciones, conceptos, valores y prácticas que constituyen un medio para ver la realidad (Harrison 2015).

Aunque en sus inicios el propósito era formalizar el modelado de arquitecturas de sistemas (Masuda y Viswanathan 2019), se han convertido en una estructura abstracta e inequívoca (Lankhorst 2009, Nardello 2019, Prayitno 2019) para clasificar, organizar y modelar (Stroud 2019) las representaciones descriptivas semánticas (Mendieta 2014) de toda la empresa (Zachman, 2003). Un marco de trabajo no es en sí una arquitectura (Selcan y Buchalcevova 2013, Schekkerman 2006), es un modelo de comunicación para desarrollar y utilizar una arquitectura (Erdenebold et al. 2019, Schekkerman 2011). Otros autores plantean un enfoque multidimensional (Mondorf y Wimmer 2017) utilizando herramientas y un vocabulario común (Bernus y Noran 2010, Open Group 2018, Rohloff 2011).

El término marco de trabajo se usa principalmente para indicar el componente central de una práctica arquitectónica (Abunadi 2019) que genera una lista de mecanismos de abstracción (Gokhale 2010), como perspectivas, puntos de vista, arquitecturas y dimensiones (Zarvic \& Wieringa, 2006). Son modelos conceptuales (Abdallah y Galal-Edeen 2009) formados por un conjunto de supuestos, conceptos, valores y prácticas que constituyen una forma de ver la realidad (Sessions 2008).
Un marco de trabajo identifica el alcance de la arquitectura y establece relaciones entre las áreas de la misma, reflejando su alcance (Hameed et al. 2010, Vargas 2015) a través de su modelo geométrico y creando un conjunto abstracto de vistas de una empresa (Bernard 2012). Cada marco tiene su propia estructura, estrategia y modelos (Sandoval, Galvez, y Moscoso 2017) y pueden ser utilizados por las organizaciones de acuerdo a sus necesidades (Qurratuaini 2017).

Existen numerosas referencias de la utilidad de los marcos de trabajo. Estos contribuyen al logro de una estructura analítica (Mondorf y Wimmer 2017) tanto para la arquitectura como para su proceso de diseño (Perroud y Inversini 2013, Foorthuis 2012). Aportan un contexto para estructurar el pensamiento organizacional (Banaeianjahromi 2018), proporcionan la base para que el hardware y el software trabajen juntos (Urbaczewski, \& Mrdalj, 2006), representan la organización fundamental de un sistema (Joubert 2010) y guían el esfuerzo operacional (Moscoso y Lujan 2017). Ofrecen además una representación conceptual (Foorthuis 2012) de todos los elementos presentes en una arquitectura empresarial instrumentados por herramientas de soporte y lenguaje unificado (Almisned 2017).

Aunque en menor medida, existen opiniones negativas acerca de los marcos de trabajo de arquitectura empresarial. Estas opiniones se centran en su proliferación desmedida y en su baja utilidad práctica. La amplia variedad de marcos de trabajo existentes (Bernus y Doumeingts, 2010, Sánchez y Collado 2017, Roach 2011, Moscoso-Zea et al. 2019, Schekkerman 2004, Yoganingrum et al. 2013, Okhrimenko 2017, Saint-Louis 2019) es producto de la evolución continua de la arquitectura empresarial. Debido a esta variedad existe un alto nivel de mutabilidad entre los marcos (Sultanow et al. 2016) y aparece de forma tácita cierta competencia entre algunos de estos (Bernus y Doumeingts 2010).

En cuanto a la naturaleza práctica de los marcos de trabajo existen cuestionamientos ya que sus prácticas individuales no presentan la capacidad de satisfacer todos los aspectos necesarios de la empresa (Noran 2018, Kotusev 2018). Las interconexiones entre las diferentes vistas pueden tornarse complejas (Essien 2019) y existe confusión para comprender el propósito y el dominio cubierto por cada modelo. Por esta razón muchas organizaciones no utilizan la arquitectura empresarial como herramienta específica en procesos de transformación exitosos (Ajer 2020). 
Para Urbaczewski, y Mrdalj (2018) el papel de los marcos de trabajo ha sido exagerado ya que no definen necesariamente la práctica de la arquitectura resultante y podría tratarse de una moda administrativa (Ajer 2020). Además muchas empresas no utilizan los marcos de trabajo o utilizan solo elementos de estos (Kotusev 2016) porque no existe una consideración clara de las bases prácticas en la aplicación de los marcos de trabajo (Abunadi 2019). Muchas organizaciones desisten en su aplicación por la obligatoriedad de personalizarlos (Kotusev 2016) y de las que logran aplicarlos muy pocas siguen los pasos prescritos por dichos marcos (Kotusev 2018). Pero no están totalmente demostrados estos planteamientos. Muchas empresas fracasan no porque el marco de trabajo adoptado no sea adecuado, sino porque solo se enfocan en el modelo y no consideran como implementar y mantener la arquitectura (Banaeianjahromi y Smolander 2017). También fracasan en la selección del marco de trabajo adecuado ya que estos varían en enfoque, alcance e intención (Mahmood y Hill 2011).

En una encuesta realizada por (Qazi et al. 2019) para analizar como los marcos de trabajo son utilizados en un grupo de empresas se demuestra que muchas de estas no tienen la capacidad de priorizar las cualidades deseadas de un marco. Muchas tampoco prestan atención a las implicaciones socio-técnicas (Scherer y Wimmer 2017) que traen consigo la aplicación, porque depende de la aceptación de las partes interesadas y de gobierno arquitectónico (Mondorf y Wimmer 2017). A pesar de esos inconvenientes en la actualidad se muestra un interés creciente en los marcos de trabajo (Laschitza y Undén 2017, Simón et al. 2013, Nilsen 2012) que siguen generando polémica en el ámbito académico y empresarial.

\subsection{Análisis de métodos de evaluación de marcos de trabajo}

En el escenario de diversidad en que operan los marcos de trabajo de arquitectura empresarial (Bui, 2017, Moscoso et al., 2019) es de esperar que existan un sinnúmero de investigaciones y publicaciones tratando de establecer pautas de evaluación, comparación y selección. En medio de este caos la elección del modelo utilizado depende de la perspectiva, porque cada uno tiene un alcance y actividades diferentes (Prayitno, 2019).

Los estudios comparativos varían mucho en su enfoque, fundamento teórico y esquemas de comparación. Algunos comparan los marcos de trabajo, otros se centran en áreas específicas de la arquitectura empresarial (Bui, 2017), mientras que otros se basan en la funcionalidad y no incluyen elementos como la facilidad de adquisición y la disponibilidad.

La cantidad y variedad de criterios utilizados por los métodos consultados es grande, pero existen muchos aspectos en común en la mayoría de ellos. En la mayoría de los casos no se justifican teóricamente los criterios (Bui, 2017). Después de la revisión de la literatura se identifican 9 métodos de evaluación y comparación de marcos de trabajo de arquitectura empresarial. Estos se muestran en la tabla 1. 
Tabla 1 Métodos de evaluación de marcos de trabajo de arquitectura empresarial.

\begin{tabular}{|c|c|c|c|c|}
\hline Id & Año & Autor & Referencia & Caracterización \\
\hline A & 2006 & Urbaczewski, \& Mrdalj & $\begin{array}{l}\text { (Urbaczewski, \& Mrdalj, } \\
\text { 2018) }\end{array}$ & $\begin{array}{l}\text { Utiliza } 3 \text { perspectivas: por vistas, por niveles de } \\
\text { abstracción y por etapas del ciclo de vida. }\end{array}$ \\
\hline B & 2006 & Leist y Zellner & (Bui, 2017) & $\begin{array}{l}\text { Utiliza elementos como el meta-modelo, el } \\
\text { procedimiento, las técnicas, el papel y el documento } \\
\text { de especificación. }\end{array}$ \\
\hline $\mathrm{C}$ & 2006 & Abdallah y Galal-Edeen & (Bui, 2017) & $\begin{array}{l}\text { Utiliza } 11 \text { criterios que van desde elementos como } \\
\text { la definición y comprensión de la arquitectura hasta } \\
\text { criterios como herramientas de visualización y el } \\
\text { diseño transaccional. }\end{array}$ \\
\hline $\mathrm{D}$ & 2008 & Roger Sessions & (Sessions, 2008) & $\begin{array}{l}\text { Presenta criterios que van desde aspectos taxonómicos } \\
\text { y conceptuales hasta aspectos de adquisición y uso. }\end{array}$ \\
\hline $\mathrm{E}$ & 2011 & Dube y Dixit & (Dube \& Dixit, 2011) & $\begin{array}{l}\text { Agrupa los criterios en tres clasificaciones: } \\
\text { por objetivos de orden superior, por soporte de } \\
\text { requerimientos no funcionales y por resultados. }\end{array}$ \\
\hline $\mathrm{F}$ & 2013 & Rouhani et al. & (Bui, 2017) & $\begin{array}{l}\text { Utiliza tres aspectos principales: conceptos, modelado } \\
\text { y proceso. }\end{array}$ \\
\hline G & 2017 & Svyatoslav Kotusev & (Kotusev, 2017) & $\begin{array}{l}\text { Analiza ítems como el enfoque esencial, los artefactos } \\
\text { de arquitectura, los términos clave, las ventajas, las } \\
\text { desventajas y la aplicabilidad. }\end{array}$ \\
\hline $\mathrm{H}$ & 2016 & Purnawan y Surendro & (Bui, 2017) & $\begin{array}{l}\text { Se comparan los marcos de trabajo mediante el uso } \\
\text { de criterios como la integralidad de la taxonomía, } \\
\text { la integridad del proceso, el enfoque comercial, la } \\
\text { orientación al gobierno, entre otros. }\end{array}$ \\
\hline I & 2017 & Nikpay y col. & (Bui, 2017) & $\begin{array}{l}\text { Analiza } 11 \text { criterios estableciendo las prioridades } \\
\text { en criterios de interoperabilidad, la gestión de los } \\
\text { requisitos de arquitectura y elementos relacionados con } \\
\text { la facilidad de uso. }\end{array}$ \\
\hline
\end{tabular}

Aunque los métodos de evaluación de los marcos de trabajo han tenido un impacto positivo también provocan confusión. La competencia entre diferentes modelos y marcos de arquitectura empresarial ha estado causando dificultades para lograr una comprensión clara del propósito principal y el dominio cubierto por cada modelo (Kimpimäki, 2014). La mayoría de los métodos solo evalúan en función de los criterios elegidos, pero interpretan de manera limitada el significado de las diferencias (Bui, 2017). Estas diferencias son muy importantes si se tiene la necesidad de elegir un marco de trabajo y no se quiere seguir estrictamente las tendencias. Existe consenso en que los resultados en la aplicación de la arquitectura empresarial dependen no solo de la eficacia de la misma sino del método de implementación utilizado. Los estudios que analizan las diferencias entre los marcos de trabajo siguen siendo principalmente informativos y descriptivos (Bui, 2017). Los enfoques actuales de evaluación no cubren todos los aspectos, no cuentan con modelos estructurados, son difíciles de entender y carecen de un método de evaluación. Existen muchos desafíos en la comparación de los marcos de trabajo de arquitectura empresarial (Urbaczewski \& Mrdalj, 2006), uno de los más importantes es lograr un criterio común en la definición de lo que es un modelo adecuado. Esta definición suele tornarse difusa en escenarios donde la selección de un marco de trabajo depende de una funcionalidad específica o de un atributo determinado previamente.

\section{Métodos utilizados en la investigación}

En la presente investigación se estudian los marcos de trabajo de arquitectura empresarial desde el análisis de su diversidad y el establecimiento de pautas de evaluación. El estudio es de tipo documental ya que se basa fundamentalmente en la valoración del contenido de un gran volumen de información. Además es exploratorio, descriptivo y evaluativo. Exploratorio porque se basa en el análisis de los marcos de trabajo desde un enfoque más amplio del que suelen presentarse este tipo de estudios. Descriptivo porque se caracterizan los marcos identificados con diferentes niveles de profundidad de acuerdo a la relevancia y disponibilidad de información. Evaluativo porque se evalúan los marcos de trabajo teniendo en cuenta una serie de criterios establecidos por un método específico desarrollado por el autor. En la figura 1 se muestra el diseño del estudio con sus fases y etapas. 
Figura 1 Diseño general de la investigación.

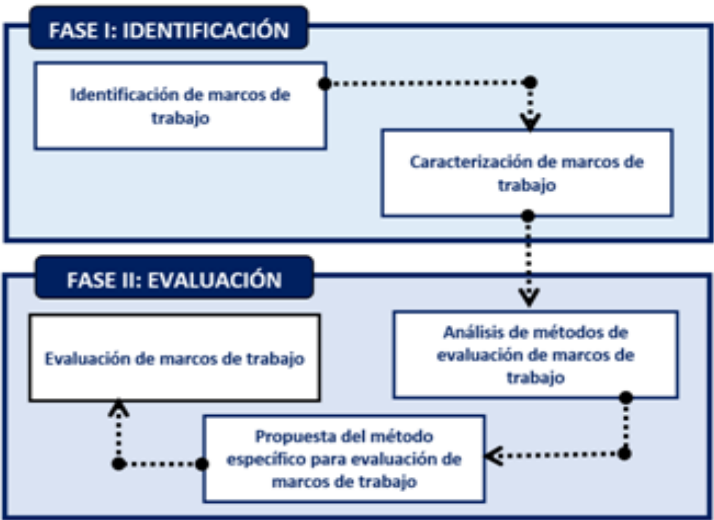

Segunda fase: Se realiza el análisis evaluativo de los marcos de trabajo identificados en el listado general. Inicialmente se estudian varios métodos de evaluación de marcos mediante la técnica de observación documental, presentación resumida y análisis crítico. De los métodos de evaluación consultados se escoge un grupo de los más relevantes. La selección se realiza escogiendo aquellos métodos que presentan una estructura clara y concreta de criterios de evaluación. Se estudia cada método seleccionado en base al listado de criterios, formas de evaluación (cualitativa o cuantitativa), evidencias de su aplicación práctica y adaptabilidad al contexto de la investigación. Cada método se analiza en base a sus potencialidades e inconvenientes, para después escoger los criterios que podrían ser relevantes para formar el método propuesto. Los principios que rigen la propuesta son los siguientes:

Integralidad: El método propuesto debe abarcar todas las dimensiones posibles para evaluar la consistencia de un marco de trabajo: funcionalidad, valor agregado potencial, ventajas, desventajas e implicaciones de su utilización.

Neutralidad: La evaluación debe referirse exclusivamente al desempeño del marco, no tiene en cuenta la historia o trayectoria del mismo. Tampoco distingue entre un marco maduro y uno emergente. El foco es como se desempeña el modelo en el criterio que se evalúa.

Cuantificación: La escala a utilizar en la propuesta debe ser cuantitativa, aunque también se acompaña por categorías cualitativas complementarias.

Documentado: La asignación de calificaciones en los criterios a considerar debe basarse en conocimiento tácito, respaldado por evidencia documental. No se admiten evaluaciones basadas en intuición o intereses personales del evaluador. ormación con un análisis descriptivo de las características del universo actual de los marcos de trabajo y con una base documental indexada sobre los modelos incluidos en el listado. 
Dinamismo: La evaluación final de los marcos de trabajo varía en el tiempo considerando nuevos hallazgos documentales, nuevos desarrollos o versiones de los modelos, nuevos casos de aplicaciones, etc. La evaluación debe actualizarse y los marcos pueden moverse vertical $\mathrm{u}$ horizontalmente en el listado bajando o subiendo en calificaciones.

Relatividad: El foco de atención no es la puntuación final o la posición del marco de trabajo en el listado. El análisis de la adecuación de un marco de trabajo por parte del usuario del método debe ser relativo en correspondencia con el objetivo práctico. Es por esto que la atención fundamental no es a la cantidad de puntos sino a como se desempeñan los marcos en determinados criterios que se consideren relevantes para una situación específica.

El método propuesto es una integración de los criterios de los métodos de referencia con la inclusión de algunos nuevos o la eliminación de otros que no aplican al contexto de la investigación. Los criterios propuestos integran los aspectos que según el autor no se pueden excluir para la evaluación integral de un marco de trabajo. A cada criterio de los métodos incluidos en el estudio se le asigna una numeración para su identificación y se realiza un análisis de correspondencia que tiene como resultado un nuevo listado de criterios propuestos. La evaluación se realiza a través de una escala likert establecida en el procedimiento. Los criterios de evaluación, así como los aspectos que evalúan se presentan a continuación:
1(Deficiente): El marco de trabajo se desempeña muy pobremente en el criterio

2(Insuficiente): El marco de trabajo se desempeña inadecuadamente en el criterio.

3(Aceptable): El marco de trabajo se desempeña bien pero debe mejorar en el criterio.

4(Satisfactorio): $\quad$ El marco se desempeña satisfactoriamenteen el criterio.

\section{Resultados y discusión}

\subsection{Identificación de marcos de trabajo de arquitectura empresarial}

La búsqueda de los marcos de trabajo potenciales se realizó abarcando una amplia base documental. Se analizaron más de 200 macos de trabajo potenciales escogiéndose un total de 160 en la propuesta final. Los marcos de trabajo rechazados en su gran mayoría no tenían claridad sobre su fecha de desarrollo y su existencia no estaba avalada documentalmente.

El listado final resumido se muestra en la tabla 2. 
Tabla 2 Listado final de marcos de trabajo de arquitectura empresarial.

\begin{tabular}{|c|c|c|c|}
\hline No & Año & Marco de Trabajo & Desarrollador \\
\hline 1 & 1980 & (IFW) IBM Información Framework & IBM (EEUU) \\
\hline 2 & 1980 & $\begin{array}{l}\text { (CAFCR) Customer Objectives, Application, } \\
\text { Functional, Conceptual, and Realization Model }\end{array}$ & $\begin{array}{cl}\text { The Embedded } & \begin{array}{l}\text { Systems Innovation Group } \\
\text { (Holanda) }\end{array}\end{array}$ \\
\hline 3 & 1984 & $\begin{array}{cl}\text { (GRAI) Graphs } & \begin{array}{l}\text { with Results and Actions } \\
\text { Interrelated }\end{array}\end{array}$ & $\begin{array}{c}\text { Laboratory of Automation and Products of } \\
\text { University Bordeaux (Francia) }\end{array}$ \\
\hline 4 & 1986 & $\begin{array}{c}\text { (PRISM) Partnership for Research in Information } \\
\text { Systems Management }\end{array}$ & Index Systems and Hammer and Company(EEUU) \\
\hline 5 & 1987 & Zachman Enterprise Architecture Framework & Zachman Inc. (EEUU) \\
\hline 6 & 1988 & $\begin{array}{c}\text { (ITIL) Information Technology Infrastructure } \\
\text { Library }\end{array}$ & $\begin{array}{l}\text { Central Computer and Telecommunications Agency } \\
\text { (CCTA) (Reino Unido) }\end{array}$ \\
\hline 7 & 1988 & (TMN) Telecommunication Management Network & $\begin{array}{l}\text { International Telecommunication Union (ITU-TS ) } \\
\text { (Multinacional) }\end{array}$ \\
\hline 8 & 1989 & (NIST-EA) NIST Enterprise Architecture Model & $\begin{array}{c}\text { National Institute of Standards and Technology } \\
\text { (NIST)(EEUU) }\end{array}$ \\
\hline 9 & 1990 & (PERA) Purdue Enterprise Reference Architecture & Purdue University (EEUU) \\
\hline 10 & 1990 & $\begin{array}{c}\text { (CIMOSA) Computer Integrated Manufacture } \\
\text { Open System Architecture }\end{array}$ & $\begin{array}{c}\text { European CIM Architecture (AMICE) y CIMOSA } \\
\text { Association (Multinacional) }\end{array}$ \\
\hline 11 & 1990 & $\begin{array}{c}\text { (GERAM) Generalized Enterprise Reference } \\
\text { Architecture and Methodology }\end{array}$ & $\begin{array}{c}\text { Task Force on Architectures for Enterprise } \\
\text { Integration (IFIP-IFAC) (EEUU) }\end{array}$ \\
\hline 12 & 1990 & $\begin{array}{c}\text { (CBDI-SAE-CBDI) Service Architecture \& } \\
\text { Engineering for SOA }\end{array}$ & CBDI Inc. (EEUU) \\
\hline 13 & 1990 & $\begin{array}{ccc}\text { (EAIF) } & \begin{array}{c}\text { Enterprise Architecture } \\
\text { Framework }\end{array} & \text { Integration } \\
\end{array}$ & Object Management Group (OMG) (EEUU) \\
\hline 14 & 1990 & (OMA) Object Management Architecture & Object Management Group (OMG) (EEUU) \\
\hline 15 & 1990 & (GERA) Generic Enterprise Reference Architecture & $\begin{array}{lll}\text { IFIP-IFAC Task } \begin{array}{l}\text { Force on Architectures for } \\
\text { EI(EEUU) }\end{array} \\
\end{array}$ \\
\hline 16 & 1990 & NIST Enterprise Architecture Model & U.S. Federal Government (EEUU) \\
\hline 17 & 1991 & $\begin{array}{cc}\text { (CORBA) Common Object Request Broker } \\
\text { Architecture }\end{array}$ & Object Management Group (OMG) (EEUU) \\
\hline 18 & 1992 & $\begin{array}{c}\text { (MINNESOTA-AEF) Minnesota Enterprise } \\
\text { Architecture } \\
\end{array}$ & $\begin{array}{c}\text { Minnesota Enterprise Architecture Development } \\
\text { Committee (EEUU) }\end{array}$ \\
\hline 19 & 1992 & $\begin{array}{c}\text { (ARIS) Architecture of Integrated Information } \\
\text { Systems }\end{array}$ & $\begin{array}{c}\text { University of Saarbrücken, IDS Scheer AG } \\
\text { (Alemania) }\end{array}$ \\
\hline 20 & 1992 & (APQC) Process Classification Framework & APQC org. (EEUU) \\
\hline 21 & 1993 & (IAF) Integrated Architecture Framework & Capgemini S.A. (Francia) \\
\hline 22 & 1994 & (MEMO) Multiperspective Enterprise Modeling & University of Duisturg-Essen (Alemania) \\
\hline 23 & 1994 & (SOM) Semantic Object Model Approach & University of Bamberg (Alemania) \\
\hline 24 & 1995 & $\begin{array}{cccc}\text { (TOGAF) The } & \begin{array}{l}\text { Open Group Architecture } \\
\text { Framework }\end{array} & \\
\end{array}$ & The Open Group (EEUU) \\
\hline 25 & 1995 & $\begin{array}{c}\text { Philippe } \begin{array}{c}\text { Kruchten's } 4+1 \text { View Model of } \\
\text { Architecture }\end{array} \\
\end{array}$ & $\begin{array}{l}\text { Philippe Kruchten (Rational Software Corporation) } \\
\text { (Multinacional) }\end{array}$ \\
\hline 26 & 1995 & SABSA Framework & $\begin{array}{l}\text { SABSA Institute } \\
\text { (Reino Unido) }\end{array}$ \\
\hline
\end{tabular}




\begin{tabular}{|c|c|c|c|}
\hline 27 & 1996 & $\begin{array}{cc}\text { (PMBOK) Project Management Body of } \\
\text { Knowledge }\end{array}$ & Project Management Institute (PMI) (EEUU) \\
\hline 28 & 1996 & (IFW) Information FrameWork & IBM (EEUU) \\
\hline 29 & 1996 & $\begin{array}{c}\text { (COBIT) Control Objectives for Information and } \\
\text { Related Technology }\end{array}$ & $\begin{array}{l}\text { Information Systems Audit and Control Association } \\
\text { (ISACA) (Multinacional) }\end{array}$ \\
\hline 30 & 1996 & (SCOR) Supply Chain Operations Reference & Supply Chain Council. (SCC) (EEUU) \\
\hline 31 & 1996 & (JTA) DoD Joint Technical Architecture & U.S. Departament of Defense (EEUU) \\
\hline 32 & 1997 & (JTA) Joint Technical Architecture & U.S. Departament of Defense (EEUU) \\
\hline 33 & 1997 & $\begin{array}{l}\text { (ARDIN) Arquitectura de Referencia para el } \\
\text { Desarrollo Integrado de la Empresa }\end{array}$ & Grupo IRIS de la Universidad Jaume I (España) \\
\hline 34 & 1997 & (UVA Model) Uniform Visualization Architecture & San Diego Super Computer Center (EEUU) \\
\hline 35 & 1997 & $\begin{array}{c}\text { (TISAF) Treasury Information System Architecture } \\
\text { Framework }\end{array}$ & U.S. Treasury Department (EEUU) \\
\hline 36 & 1998 & (EAP) Enterprise Architecture Planning & Steven H. Spewak (EEUU) \\
\hline 37 & 1998 & $\begin{array}{c}\text { (PPOOA) Processes Pipelines in Object Oriented } \\
\text { Architectures }\end{array}$ & Universidad Politécnica de Madrid (España) \\
\hline 38 & 1998 & $\begin{array}{l}\text { (RM-ODP) ISO Reference Model for Open } \\
\text { Distributed Processing }\end{array}$ & $\begin{array}{ll}\text { International } & \text { Organization for Standardization } \\
& \text { (ISO) (Multinacional) }\end{array}$ \\
\hline 39 & 1999 & $\begin{array}{l}\text { (C4ISR) Command, Control, Communications, } \\
\text { Computers, Intelligence, Surveillance and } \\
\text { Reconnaissance }\end{array}$ & U.S. Departament of Defense (EEUU) \\
\hline 40 & 1999 & $\begin{array}{l}\text { (MACCIS) An Architecture Description } \\
\text { Framework for Technical Info Structures and their } \\
\text { Enterprise Environment }\end{array}$ & $\begin{array}{c}\text { Norwegian Defence Logistics Organisation (NDLO) } \\
\text { (Noruega) }\end{array}$ \\
\hline 41 & 1999 & (IADS) IBM Architecture Description Standard & IBM(EEUU) \\
\hline 42 & 1999 & $\begin{array}{l}\text { Integración } \quad \text { Empresarial-Gestión } \\
\text { Integrada de Procesos }\end{array}$ & Universidad Politécnica de Valencia (España) \\
\hline 43 & 1999 & (FEAF) Federal Enterprise Architecture Framework & $\begin{array}{c}\text { U.S Federal Government. Federal CIO Council } \\
\text { (EEUU) }\end{array}$ \\
\hline 44 & 2000 & $\begin{array}{c}\text { (TEAF) Treasury Enterprise Architecture } \\
\text { Framework }\end{array}$ & U.S. Treasury Department (EEUU) \\
\hline 45 & 2000 & $\begin{array}{l}\text { (Doc-Framework) Framework para Documentar } \\
\text { Frameworks }\end{array}$ & Universidad de La Plata (Argentina) \\
\hline 46 & 2000 & (ADO) Australian Defence Organisation & $\begin{array}{ccc}\text { (DTSO) Defense Science and Technology } \\
\text { Organization (Australia) }\end{array}$ \\
\hline 47 & 2000 & $\begin{array}{ll}\text { (SAGA) Standards and Architecture for } \\
\text { e-government Applications }\end{array}$ & $\begin{array}{c}\text { The German Commission of the Federal } \\
\text { Government for IT (Alemania) }\end{array}$ \\
\hline 48 & 2001 & $\begin{array}{l}\text { (AGATE) Atelier de Gestion de l'Architecture des } \\
\text { Systemes d'Information et de Communication }\end{array}$ & $\begin{array}{c}\text { Délégation Générale pour l'Armement (DGA) } \\
\text { (Francia) }\end{array}$ \\
\hline 49 & 2001 & (GEA) Government Enterprise Architecture & $\begin{array}{l}\text { Queensland Government Office Chief Information } \\
\text { (QGCIO) (Australia) }\end{array}$ \\
\hline 50 & 2001 & $\begin{array}{c}\text { (eTOM) Enhanced Telecommunication Operations } \\
\text { Map }\end{array}$ & Telemagement Forum(Multinacional) \\
\hline 51 & 2001 & (SAM) Solution Architecting Mechanism & $\begin{array}{l}\text { School of Computer and Communication Sciences } \\
\text { (IC) (EEUU) }\end{array}$ \\
\hline 52 & 2001 & $\begin{array}{c}\text { (DYA) Dynamic Architecture for Modelling and } \\
\text { Development }\end{array}$ & The Consulting Company Sogeti (Holanda) \\
\hline 53 & 2001 & (EAG) The MIT Enterprise Architecture Guide & $\begin{array}{cc}\text { Massachusetts Institute of Technology (MIT) } \\
\text { (EEUU) }\end{array}$ \\
\hline
\end{tabular}




\begin{tabular}{|c|c|c|c|}
\hline 54 & 2001 & $\begin{array}{c}\text { (DNDAF) Canadian Armed Forces Architecture } \\
\text { Framework }\end{array}$ & $\begin{array}{c}\text { Department of National Defense Canadian Armed } \\
\text { Forces (Canada) }\end{array}$ \\
\hline 55 & 2001 & (WAAF) Web Application Architecture Framework & The University of Sydney (Australia) \\
\hline 56 & 2001 & $\begin{array}{c}\text { (E2AF) Extended Enterprise Architecture } \\
\text { Framework }\end{array}$ & $\begin{array}{l}\text { Institute for Enterprise Architecture Developments } \\
\text { (IFEAD)(EEUU) }\end{array}$ \\
\hline 57 & 2002 & (EXAF) Extreme Architecture Framework & Idea Group Inc. (Multinacional) \\
\hline 58 & 2002 & (VCOR) Value Chain Operations Reference Model & $\begin{array}{l}\text { Supply Chain Council and the Value Chain Group } \\
\text { (VCG)(EEUU) }\end{array}$ \\
\hline 59 & 2002 & (LESAT) Lean Enterprise Self-Assessment Tool & $\begin{array}{cc}\text { Massachusetts Institute of Technology (MIT) } \\
\text { (EEUU) }\end{array}$ \\
\hline 60 & 2002 & $\begin{array}{c}\text { (QGEA) Queensland Government Enterprise } \\
\text { Architecture }\end{array}$ & Queensland Government of Australia (Australia) \\
\hline 61 & 2003 & ARCHIMATE Framework & $\begin{array}{l}\text { Telematica Institute / Novay } \\
\text { (Holanda) }\end{array}$ \\
\hline
\end{tabular}

\begin{tabular}{|c|c|c|c|}
\hline 62 & 2003 & $\begin{array}{c}\text { (DoDAF) Department of Defense Architecture } \\
\text { Framework }\end{array}$ & U.S. Departament of Defense (EEUU) \\
\hline 63 & 2003 & $\begin{array}{ccc}\text { (AF-EAF) Air Force Enterprise Architecture } \\
\\
\text { Framework }\end{array}$ & U.S. Departament of Defense (EEUU) \\
\hline 64 & 2003 & (LEAP) LJMU Enterprise Architecture Pilot & Liverpool John Moores University (Reino Unido) \\
\hline 65 & 2003 & (xAF) Extensible Architecture Framework & Radboud University (Holanda) \\
\hline 66 & 2003 & (EITA) The SKF Enterprise IT Architecture & SKF Group(EEUU) \\
\hline 67 & 2003 & (NASCIO) Enterprise Architecture Maturity Model & $\begin{array}{c}\text { National Association of State Chief Information } \\
\text { Officers (EEUU) }\end{array}$ \\
\hline 68 & 2003 & $\begin{array}{cc}\text { (SEAM) Systemic Enterprise Architecture } \\
\text { Methodology }\end{array}$ & $\begin{array}{l}\text { École Polytechnique Fédérale de Lausanne (EPFL) } \\
\text { (Suiza) }\end{array}$ \\
\hline 69 & 2003 & The EA management approach of MIT & $\begin{array}{l}\text { MIT Sloan Center for Information Systems Research } \\
\qquad(\text { CISR)(EEUU) }\end{array}$ \\
\hline 70 & 1993 & (EIF) European Interoperability & Union Europea (Multinacional) \\
\hline 71 & 2003 & The EA management approach of TU Lisbon & TU Lisbon (Portugal) \\
\hline 72 & 2003 & NCOW Reference Model & U.S. Departament of Defense (EEUU) \\
\hline 73 & 2003 & (IRS) Internet Reasoning Service & Knowledge Media Institute KMI (Reino Unido) \\
\hline 74 & 2004 & (FEAR) Finnish Enterprise Architecture Research & University of Jyväskylä (Finlandia) \\
\hline 75 & 2004 & (NATO) The NATO Architecture Framework & U.S. Departament of Defense (EEUU) \\
\hline 76 & 2004 & $\begin{array}{ccc}\text { (EABOK) Enterprise Architecture Body } \\
\text { Knowledge }\end{array}$ & $\begin{array}{c}\text { MITRE's Center for Innovative Computing and } \\
\text { Informatics(EEUU) }\end{array}$ \\
\hline 77 & 2004 & (BTEP) GC Enterprise Architecture and Standards & Treasury Board of Canada Secretariat (Canada) \\
\hline 78 & 2004 & $\begin{array}{c}\text { (CAFCR) Multi-view Method for Embedded } \\
\text { Systems Architecting }\end{array}$ & Gaudí Systems Architecting (Holanda) \\
\hline 79 & 2004 & $\begin{array}{c}\text { (DHSF) Department of Homeland Security } \\
\text { Enterprise Architecture }\end{array}$ & Department of Homeland Security (EEUU) \\
\hline 80 & 2004 & $\begin{array}{c}\text { (BEAM) Business Enterprise Architecture } \\
\text { Modeling }\end{array}$ & $\begin{array}{cl}\text { Ken Orr Institute. Kansas Department of } \\
\text { Transportation (Alemania) }\end{array}$ \\
\hline 81 & 2004 & The EA management approach of KTH Stockholm & KTH Stockholm University (Suiza) \\
\hline
\end{tabular}




\begin{tabular}{|c|c|c|c|}
\hline 82 & 2004 & (BEAMS) Building blocks for Enterprise & TU Munich(Alemania) \\
\hline \multicolumn{4}{|c|}{ Architecture Management Solutions } \\
\hline 83 & 2004 & (JFMIP) Federal Financial Management System & U.S. Treasury Department (EEUU) \\
\hline 84 & 2004 & $\begin{array}{l}\text { (FADE) The Framework for the Architectural } \\
\text { Development of the Enterprise }\end{array}$ & IFIP-IFAC Task Force(EEUU) \\
\hline 85 & 2005 & Gartner Enterprise Architectural Framework & Gartner Inc. (EEUU) \\
\hline 86 & 2005 & (MEA) State of Minnesota Enterprise Architecture & Minnesota Office of Enterprise Technology (EEUU) \\
\hline 87 & 2005 & FDIC Enterprise Architecture Framework & $\begin{array}{c}\text { Federal Deposit Insurance Corporation (FDIC) } \\
\text { (EEUU) }\end{array}$ \\
\hline 88 & 2005 & EA3 Cube Framework & Scott A. Bernard, (Syracuse University)(EEUU) \\
\hline 88 & 2005 & EA3 Cube Framework & Scott A. Bernard, (Syracuse University)(EEUU) \\
\hline 89 & 2005 & (MDA) The Model Driven Architecture & OMG Group (EEUU) \\
\hline 90 & 2006 & SAP Enterprise Architecture Frameworks & Equipo SAP EA (Alemania) \\
\hline 91 & 2007 & (OIO EA) OIO Enterprise Architecture Method & $\begin{array}{l}\text { Ministry of Science, Technology and Innovation } \\
\text { (EEUU) }\end{array}$ \\
\hline 92 & 2006 & $\begin{array}{c}\text { (JRA) The Justice Reference Architecture } \\
\text { Specification }\end{array}$ & U.S. Department of Justice (DOJ) (EEUU) \\
\hline 93 & 2006 & $\begin{array}{c}\text { (MITA) The Monash Information Technology } \\
\text { Architecture }\end{array}$ & Monash University (EEUU) \\
\hline 94 & 2006 & $\begin{array}{cc}(\text { EAFoC) Enterprise Architecture Framework } \\
\text { Based on Commonality }\end{array}$ & $\begin{array}{l}\text { Korea University and Sang-Ju National University } \\
\text { (Korea) }\end{array}$ \\
\hline 95 & 2006 & $\begin{array}{l}\text { (NASEAF) National Airspace System Enterprise } \\
\text { Architecture }\end{array}$ & FAA (Administración Federal de Aviación) (EEUU) \\
\hline 96 & 2006 & $\begin{array}{l}\text { (SOA-RM) OASIS Reference Model for Service } \\
\text { Oriented Architecture }\end{array}$ & Technical Committee (TC) (EEUU) \\
\hline 97 & 2006 & (dAF) Domain-specific Architecture Framework & Technische Universitat Munchen (Alemania) \\
\hline 98 & 2007 & (DRA1) Dragon 1 Framework & Dragon1 Architecture Foundation (Holanda) \\
\hline 99 & 2007 & HHS Enterprise Architecture & Department of Health and Human Services (EEUU) \\
\hline 100 & 2007 & $\begin{array}{c}\text { (EAAF) Enterprise Architecture Assessment } \\
\text { Framework }\end{array}$ & $\begin{array}{l}\text { The Office of Management and Budget (OMB) } \\
\text { (EEUU) }\end{array}$ \\
\hline 101 & 2007 & $\begin{array}{cc}\text { (SGCAF) Smart Grid Conceptual Architecture } \\
\text { Framework }\end{array}$ & $\begin{array}{l}\text { National Institute Of Standards and Technology } \\
\text { (NIST) (EEUU) }\end{array}$ \\
\hline 102 & 2007 & (OIOAF) OIO Enterprise Architecture Method & $\begin{array}{l}\text { Ministry of Science, Technology and Innovation } \\
\text { (EEUU) }\end{array}$ \\
\hline 103 & 2007 & $\begin{array}{ccc}\text { (COSMO) Conceptual Service } & \text { Modelling } \\
\text { Framework }\end{array}$ & University of Twente (Holanda) \\
\hline 105 & 2007 & $\begin{array}{l}\text { The EA Management approach of the University } \\
\text { of St. Gallen }\end{array}$ & University of St. Gallen (Suiza) \\
\hline 106 & 2007 & (BMM) Business Motivation Model & $\begin{array}{l}\text { Object Management Group } \\
\text { (EEUU) }\end{array}$ \\
\hline 107 & 2007 & $\begin{array}{c}\text { (ARCON) Reference architecture for integrated } \\
\text { knowledge networks }\end{array}$ & Stellenbosch University (Sudafrica) \\
\hline 108 & 2008 & (OEAF) Oracle Enterprise Architecture Framework & Oracle Corporation (EEUU) \\
\hline 109 & 2008 & $\begin{array}{c}\text { (PEAF) Pragmatic Enterprise Architecture } \\
\text { Framework }\end{array}$ & U.S. Federal Government(EEUU) \\
\hline
\end{tabular}




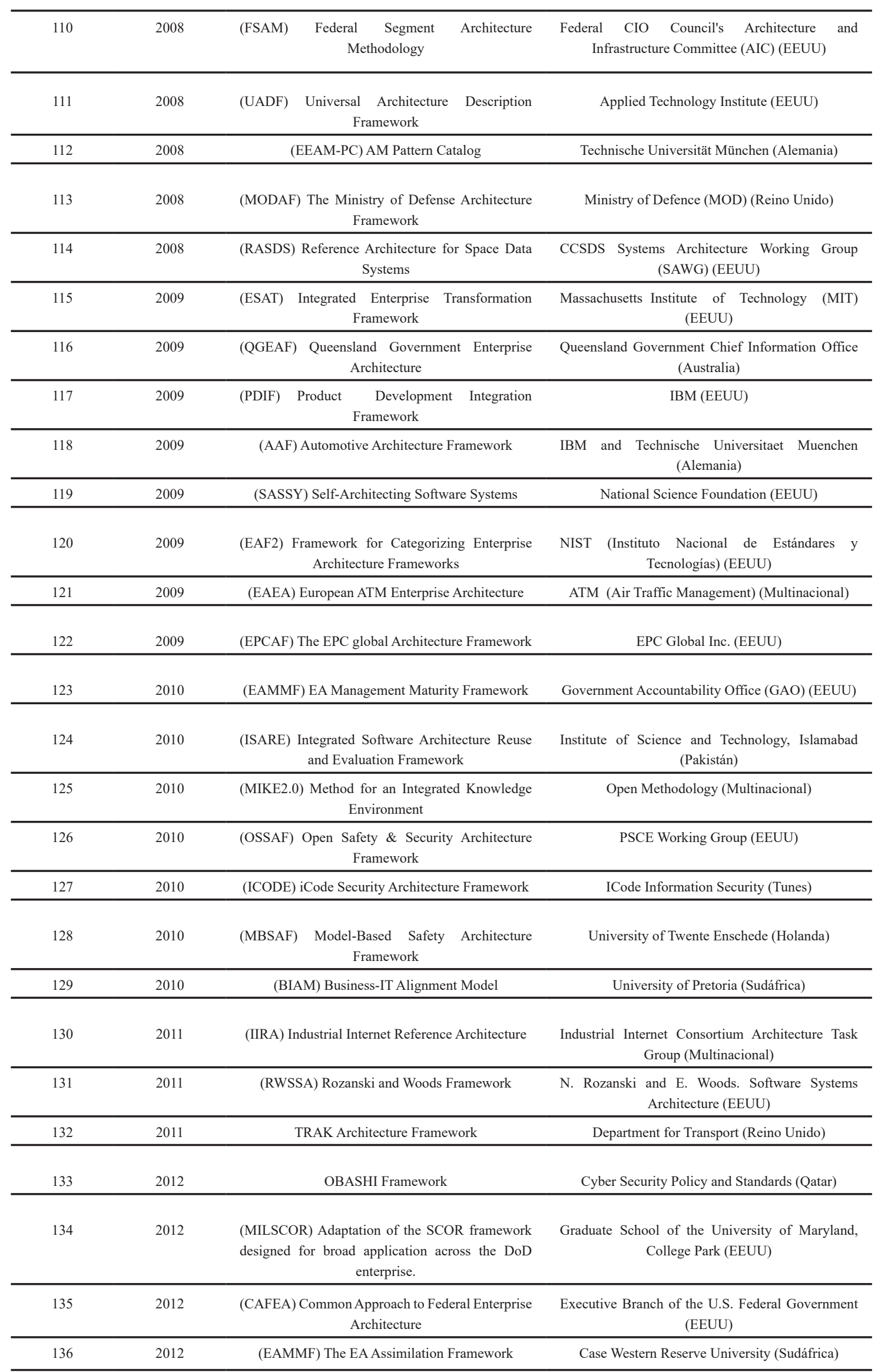




\begin{tabular}{|c|c|c|c|}
\hline 137 & 2012 & $\begin{array}{c}\text { (AFE) Architecting the Future Enterprise } \\
\text { Framework }\end{array}$ & Instituto Politécnico Naval (Chile) \\
\hline 138 & 2012 & $\begin{array}{c}\text { (UAF) Technology Update on the Unified } \\
\text { Architecture }\end{array}$ & OTAN (Multinacional) \\
\hline 139 & 2013 & (EAAT) Enterprise Architecture Analysis Tool & Royal Institute of Technology Stockholm (Suecia) \\
\hline 140 & 2013 & $\begin{array}{c}\text { (SOVO-CM) Change Management in Service } \\
\text { Oriented Virtual Organizations }\end{array}$ & University of Ottawa (Canada) \\
\hline 141 & 2013 & (BDAF) Big Data Architecture Framework & University of Amsterdam (Holanda) \\
\hline 142 & 2013 & 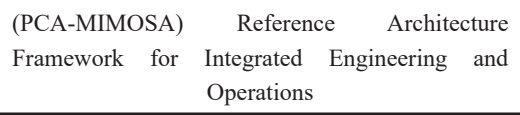 & $\begin{array}{c}\text { POSC Caesar Association (PCA) and MIMOSA } \\
\text { (EEUU) }\end{array}$ \\
\hline 143 & 2013 & $\begin{array}{c}\text { (AESS) The Adaptive Enterprise Architecture } \\
\text { Framework }\end{array}$ & University of Technology Sydney (Australia) \\
\hline 144 & 2013 & $\begin{array}{c}\text { (AGA) Australian Government Architecture } \\
\text { Reference Models }\end{array}$ & Australian Government (Australia) \\
\hline 145 & 2014 & $\begin{array}{ccc}\text { (VERAM) Virtual Enterprise } & \text { Reference } \\
& \text { Architecture }\end{array}$ & Technical University of Denmark (Dinamarca) \\
\hline 146 & 2014 & $\begin{array}{l}\text { (BplSoa) Framework para el Desarrollo de Líneas } \\
\text { de Procesos de Negocios Orientadas a Servicios }\end{array}$ & Universidad Nacional de Colombia (Colombia) \\
\hline 147 & 2014 & California Enterprise Architecture Framework & $\begin{array}{ccc}\text { Departament } & \text { of Technology of California } \\
& \text { Government (EEUU) }\end{array}$ \\
\hline 148 & 2014 & $\begin{array}{c}\text { (BIRA) Business Intelligence } \text { Reference } \\
\text { Architecture. California Enterprise Architecture } \\
\text { Framework }\end{array}$ & California Department of Technology(EEUU) \\
\hline 149 & 2015 & $\begin{array}{lll}\text { (FIEAF) Fast Implementation } & \text { Enterprise } \\
& \text { Architecture Framework }\end{array}$ & $\begin{array}{c}\text { Universidad católica de Santiago de Guayaquil } \\
\text { (Ecuador) }\end{array}$ \\
\hline 150 & 2015 & $\begin{array}{c}\text { (BAAM) Extendibility of a Proposed Business } \\
\text { Architecture Assessment Model }\end{array}$ & University of the Western Cape (Sudáfrica) \\
\hline 151 & 2016 & (BPAM) Business Process Alignment & University of the Western Cape (Sudáfrica) \\
\hline 152 & 2016 & Business Strategy and Innovation Framework & Industrial Internet Consortium (Multinacional) \\
\hline 153 & 2016 & (TRM) Oregon Technology Reference Model & Chief Information. Office of de State (EEUU) \\
\hline 154 & 2016 & $\begin{array}{c}\text { (HEAFs) Holistic Enterprise Architecture } \\
\text { Frameworks }\end{array}$ & Naif Aljlayel (Arabia Saudita) \\
\hline 155 & 2017 & $\begin{array}{c}\text { (EAMPC) Enterprise Architecture Management } \\
\text { Pattern Catalog }\end{array}$ & King's College London (Reino Unido) \\
\hline 156 & 2017 & $\begin{array}{c}\text { (SCORE) Driven Approach for Enterprise } \\
\text { Architecture }\end{array}$ & National Institute of Technology of Durgapur (India) \\
\hline 157 & 2017 & (INEAF) Iran's National EA Framework & Shahid Beheshti University (Iran) \\
\hline 158 & 2018 & (AIDAF) Adaptive Integrated Digital Architecture & Keio University (Japón) \\
\hline 159 & 2018 & (D2F) Digital Diamond EA Framework & Aalen University (Alemania) \\
\hline 160 & 2019 & $\begin{array}{c}\text { (VEEAF) } \\
\text { Architecture }\end{array}$ & Universitas Negeri Jakarta (Alemania) \\
\hline
\end{tabular}


A continuación se analiza el listado de marcos de trabajo de la tabla 2.
Por sector: Existen marcos de trabajo para los más diversos sectores e industrias y un grupo amplio son genéricos, o sea, pueden ser aplicados en cualquier contexto. En la tabla 3 se muestra la composición del listado de marcos según sector de procedencia.
Tabla 3 Marcos de trabajo por sector de procedencia.

\begin{tabular}{ccc}
\hline Sector & Cantidad & $\%$ \\
\hline Genérico & 44 & 27,50 \\
\hline Sector Especifico & 116 & 72,50 \\
\hline TOTALES & $\mathbf{1 6 0}$ & $\mathbf{1 0 0 , 0 0}$ \\
\hline
\end{tabular}

mayor cantidad de marcos pertenecen a sectores específicos. De estos el 35,59\% han sido desarrollados para las tecnologías de la información e internet. Otros sectores que sobresalen son gobierno y defensa con $27,12 \%$ y $14,41 \%$ respectivamente. Aunque en menor medida existen muchos sectores para los cuales han sido desarrollados marcos de trabajo como es el caso de las telecomunicaciones, logística, servicios financieros, educación superior y servicios de salud.

Según región: Existen marcos de trabajo en muchas regiones del mundo. En Norteamérica se encuentra la mayor proporción siendo Estados Unidos el país con más presencia de marcos con 82 para un 51,25 \%. En Europa también se han desarrollado una gran cantidad. Países como Reino Unido, Holanda y Alemania sobresalen con el $66,67 \%$ de todos los marcos desarrollados en esa región. En otras regiones como África, Asia y Latinoamérica el desarrollo ha sido menor, mientras que países como Australia han tenido un desarrollo importante en los últimos años con 7 marcos de trabajo.

Según fecha: El primer marco de trabajo referenciado en el listado es del 1980. A partir de ahí se han desarrollado modelos durante 4 décadas de manera ininterrumpida, aunque la intensidad no es la misma. En el gráfico 2 se muestra la cantidad de marcos desarrollados por fecha.

Figura 2 Proporción de marcos de trabajo por fecha.

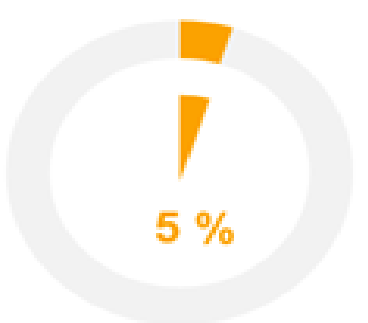

De 1980 a 1989

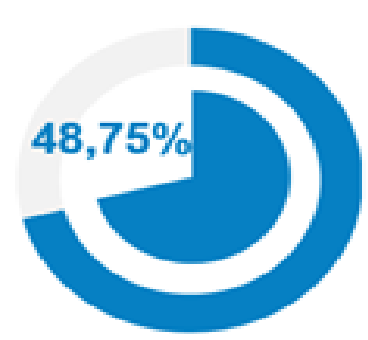

De 2000 a 2009

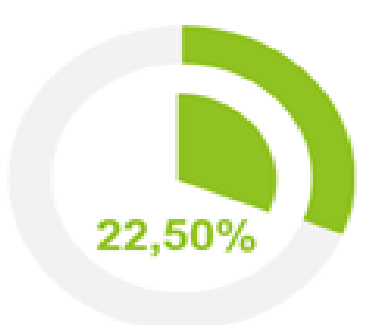

De 1990 a 1999

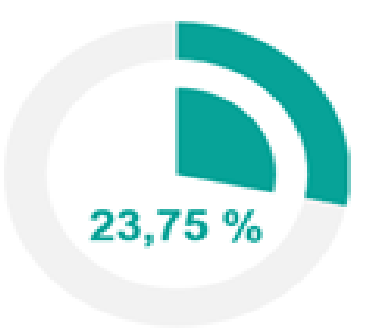

De 2010 a 2019 


\subsection{Propuesta de método de evaluación de marcos de trabajo}

La propuesta se conforma de la superposición de los criterios de los 9 métodos presentados en la tabla 1. Para facilitar el análisis se asigna una letra identificando cada método de evaluación según se presenta a continuación: (A) Urbaczewski, \& Mrdalj, (B) Leist y Zellner, (C) Abdallah y Galal-Edeen, (D) Roger Sessions, (E) Dube y Dixit, (F) Rouhani et al., (G) Svyatoslav Kotusev, (H) Purnawan y Surendro, (I) Nikpay y Col.
En la celda de intersección entre los criterios propuestos y los métodos de referencia se presentan los criterios identificados por su número. En el caso de que no exista relación entre criterios se sombrea la celda. Todos los criterios al menos tienen relación con un criterio de los métodos base excepto el P13.

En la tabla 4 se muestran los criterios de la propuesta y la relación con los criterios base.
Tabla 4 Correspondencia entre los criterios del método propuesto y las metodologías de referencia.

\begin{tabular}{|c|c|c|c|c|c|c|c|c|c|}
\hline \multirow{2}{*}{ Propuesta } & \multicolumn{9}{|c|}{ Métodos de referencia } \\
\hline & $\mathbf{A}$ & B & $\mathbf{C}$ & D & $\mathbf{E}$ & $\mathbf{F}$ & G & $\mathbf{H}$ & I \\
\hline \multicolumn{10}{|c|}{ FILTRO No 1} \\
\hline $\begin{array}{l}\text { P1. Existencia de un meta- } \\
\text { modelo }\end{array}$ & $\mathrm{X}$ & $1-5$ & $\mathrm{X}$ & $\mathrm{X}$ & 24 & $\mathrm{X}$ & $\mathrm{X}$ & $\mathrm{X}$ & $\mathrm{X}$ \\
\hline $\begin{array}{l}\text { P2. Existencia de documentación } \\
\text { de especificaciones }\end{array}$ & $\mathrm{X}$ & $\mathrm{X}$ & $\mathrm{X}$ & 8 & $6-49$ & $\mathrm{X}$ & $\mathrm{X}$ & 7 & $\mathrm{X}$ \\
\hline \multicolumn{10}{|c|}{ FILTRO No 2} \\
\hline \multicolumn{10}{|c|}{ Dimensión 1: Criterios estructurales, conceptuales y de diseño. } \\
\hline $\begin{array}{l}\text { P3. Integridad taxonómica y } \\
\text { semántica }\end{array}$ & $1-2$ & $\mathrm{X}$ & 1 & 1 & $1-4-7-15-22$ & 1 & $1-3$ & 1 & $\mathrm{X}$ \\
\hline P4. Integridad de procesos & 3 & $\mathrm{X}$ & $2-8$ & 2 & $2-3-9$ & 3 & 2 & 2 & 2 \\
\hline P5. Modelo de referencia & $\mathrm{X}$ & $\mathrm{X}$ & $4-3$ & 3 & $8-5-20$ & $\mathrm{X}$ & $\mathrm{X}$ & $\mathrm{X}$ & 11 \\
\hline $\begin{array}{l}\text { P6. Existencia de metodologías y } \\
\text { procedimientos }\end{array}$ & $\mathrm{X}$ & $2-3$ & $\mathrm{X}$ & $\mathrm{X}$ & 57 & $\mathrm{X}$ & $\mathrm{X}$ & $\mathrm{X}$ & 6 \\
\hline P7. Modelo de control y madurez & $\mathrm{X}$ & $\mathrm{X}$ & 9 & 5 & $\begin{array}{c}10-13-19-39- \\
25-26\end{array}$ & $\mathrm{X}$ & $\mathrm{X}$ & $\mathrm{X}$ & $\mathrm{X}$ \\
\hline $\begin{array}{l}\text { P8. Enfoque en el negocio, la } \\
\text { estrategia y las ventas }\end{array}$ & $\mathrm{X}$ & 4 & $5-7$ & 6 & $\begin{array}{c}11-14-46-48- \\
51-52\end{array}$ & $\mathrm{X}$ & $\mathrm{X}$ & $3-10$ & $\mathrm{X}$ \\
\hline P9. Enfoque a la partición & $\mathrm{X}$ & $\mathrm{X}$ & $\mathrm{X}$ & 7 & $\mathrm{X}$ & $\mathrm{X}$ & $\mathrm{X}$ & 5 & I8 \\
\hline P10. Enfoque al gobierno & $\mathrm{X}$ & $\mathrm{X}$ & $\mathrm{X}$ & 9 & $\mathrm{X}$ & $\mathrm{X}$ & $\mathrm{X}$ & 4 & I9 \\
\hline $\begin{array}{l}\text { P11. Descripción de aplicación y } \\
\text { catálogo }\end{array}$ & $\mathrm{X}$ & $\mathrm{X}$ & $\mathrm{X}$ & 10 & $\mathrm{X}$ & $\mathrm{X}$ & $\mathrm{X}$ & $\mathrm{X}$ & 5 \\
\hline $\begin{array}{l}\text { P12. Orientación al mejoramiento } \\
\text { de los procesos }\end{array}$ & $\mathrm{X}$ & $\mathrm{X}$ & 6 & $\mathrm{X}$ & $\mathrm{X}$ & $\mathrm{X}$ & $\mathrm{X}$ & $\mathrm{X}$ & 1 \\
\hline $\begin{array}{l}\text { P13. Determinación de roles y } \\
\text { responsabilidades }\end{array}$ & $\mathrm{X}$ & $\mathrm{X}$ & $\mathrm{X}$ & $\mathrm{X}$ & $\mathrm{X}$ & $\mathrm{X}$ & $\mathrm{X}$ & $\mathrm{X}$ & $\mathrm{X}$ \\
\hline \multicolumn{10}{|c|}{ Dimensión 2: Criterios de manejo y operación. } \\
\hline $\begin{array}{l}\text { P14. Adaptabilidad y facilidad de } \\
\text { modelado }\end{array}$ & $\mathrm{X}$ & $\mathrm{X}$ & $\mathrm{X}$ & 4 & 27 & 2 & $\mathrm{X}$ & 6 & 12 \\
\hline $\begin{array}{l}\text { P15. Coherencia, consistencia y } \\
\text { acoplamiento }\end{array}$ & $\mathrm{X}$ & $\mathrm{X}$ & $\mathrm{X}$ & $\mathrm{X}$ & $29-32-33$ & $\mathrm{X}$ & $\mathrm{X}$ & $\mathrm{X}$ & 7 \\
\hline
\end{tabular}




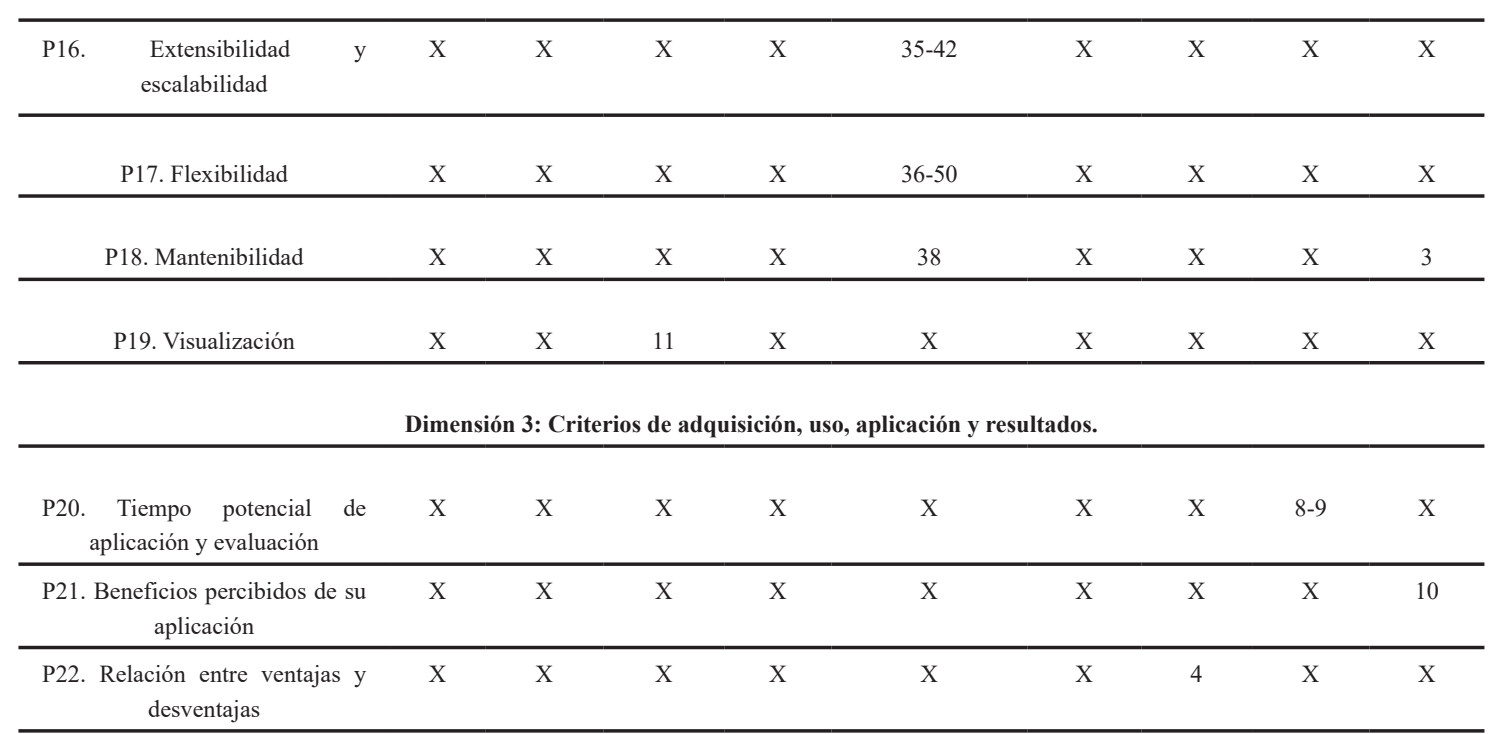

La propuesta final cuenta con 22 criterios agrupados en dos filtros de análisis.

Filtro 1. Tiene el objetivo de reducir el listado inicial de acuerdo a dos criterios generales y se basa en la existencia de información documentada. La evaluación se realiza seleccionado entre dos opciones (SI/NO) y solo pasan el filtro aquellos marcos de trabajo que sean evaluados afirmativamente en los dos criterios.

Filtro 2. Se establece en base a 20 criterios agrupados en tres dimensiones:

Criterios estructurales, conceptuales y de diseño: Establecen la consistencia estructural y de diseño del marco de trabajo. Incluye 10 criterios que valoran como el diseño y la estructura del marco responde a las necesidades de la arquitectura. Son criterios altamente funcionales y expresan como se desempeña el marco en áreas clave como es el gobierno, los procesos y el negocio.

Criterios de manejo y operación: Establecen los parámetros imprescindibles que debe cumplir el marco de trabajo para su desarrollo y ejecución. Se incluyen 9 criterios que representan aspectos como la flexibilidad, visualización, adaptabilidad, entre otros.

Criterios de adquisición, uso, aplicación y resultados: Esta dimensión es la menos tratada por los métodos que sirven de referencia al estudio. Se establecen los criterios que evalúan la accesibilidad del marco de trabajo y la facilidad con que se obtienen sus especificaciones. Se incluyen 3 criterios. En la tabla 5 se muestran los criterios del método propuesto con explicaciones resumidas sobre su significado. 
Tabla 5 Descripción de los criterios del método de evaluación propuesto.

\begin{tabular}{lccll}
\hline No & CRITERIO & SIGNIFICADO \\
\hline P1 & $\begin{array}{c}\text { Existencia de un } \\
\text { modelo }\end{array}$ & meta- & $\begin{array}{l}\text { Disponibilidad de las construcciones para la creación de modelos. Evalúa el grado en que es } \\
\text { accesible la estructura de las vistas de arquitectura y sus descripciones. }\end{array}$ \\
\hline P2 & $\begin{array}{c}\text { Documentación } \\
\text { especificaciones }\end{array}$ & de & $\begin{array}{l}\text { Se refiere a la disponibilidad de información sobre el marco de trabajo que puede estar accesible } \\
\text { mediante manuales, descripciones, estándares, etc. }\end{array}$ \\
\hline
\end{tabular}

Dimensión No 1: Criterios estructurales, conceptuales y de diseño.

P3 Integridad taxonómica y Es el grado en el que se puede utilizar el marco de trabajo para clasificar los artefactos. Trata de semántica cómo se determinan los aspectos, vistas y puntos de vista que componen la base de segmentos de la arquitectura. Describe la terminología y directrices.

P4 Integridad de procesos

Evalúa como paso a paso se describe el proceso la creación de una arquitectura empresarial manteniendo la trazabilidad. Evalúa como se guía la construcción de la arquitectura gestionando los procesos y mecanismos, garantizando una base de información, abarcando la adopción de estrategias y modelos de referencia.

\begin{tabular}{cccc}
\hline P5 & Modelo de referencia & $\begin{array}{l}\text { Utilidad de la metodología para construir un conjunto de modelos de referencia. Evalúa como } \\
\text { representa el marco en términos de modelos de análisis y diseño cumpliendo con las normas y } \\
\text { especificaciones. Analiza la existencia de repositorios o soportes de información y como se } \\
\text { describen las entidades lógicas y sus componentes. }\end{array}$ \\
\hline P6 & $\begin{array}{l}\text { Existencia de metodologías y } \\
\text { procedimientos }\end{array}$ & $\begin{array}{l}\text { Existencia de una metodología, procedimiento o método que guíe el desarrollo de la arquitectura. } \\
\text { También se tiene en cuenta la existencia de técnicas y herramientas para el desarrollo de la } \\
\text { arquitectura. }\end{array}$ \\
\hline
\end{tabular}

P7 Modelo de control y madurez Capacidad para la evaluación de la eficacia y la madurez en el uso de la arquitectura empresarial Tiene en cuenta procesos que guían la gestión y control de las arquitecturas empresariales. Analiza cómo define criterios de pruebas de conformidad de la implementación.

P8 Enfoque en el negocio, la Capacidad de utilización de la tecnología para impulsar el valor del negocio. Como se ocupa de estrategia y las ventas la identificación, la gestión y los activos en función de las necesidades de las partes interesadas. Analiza cómo se incorpora el perfil estratégico y operativo gestionando los objetivos del negocio, dirección, principios, y estrategias.

P9 Enfoque a la partición. Grado en que el marco hace posible la división en particiones autónomas de la empresa para la gestión de la complejidad. Se trata de una base de información que lleva un registro identificado de segmentos arquitectónicos.

P10 Enfoque al gobierno Utilidad del marco de trabajo en la comprensión y la creación de un modelo de gestión eficaz para la arquitectura y la gobernabilidad. Evalúa la prioridad que recibe la gobernabilidad de la arquitectura y sus diversos componentes en el marco.

P11 Descripción de aplicación y Grado en que es viable la creación de un catálogo de los bienes arquitectónicos que se pueden catálogo reutilizar en actividades futuras. Evalúa como se tienen en cuenta los requisitos de la arquitectura objetivo durante todo el proceso de aplicación de la misma.

P12 Orientación al mejoramiento Evalúa como los modelos resultantes de la arquitectura facilitan y conducen a la mejora de los de los procesos procesos que representan. Tiene en cuenta como es capaz de integrar la tecnología, la arquitectura futura, la interoperabilidad y las normas.

P13 Determinación de roles y Tiene en cuenta cómo y con la profundidad con que se identifican los roles y responsabilidades de responsabilidades cada persona o proceso dentro de la arquitectura.

Dimensión No 2: Criterios de manejo y operación

\begin{tabular}{|c|c|c|}
\hline & & Dimensión No 2: Criterios de manejo y operación \\
\hline P14 & $\begin{array}{l}\text { Adaptabilidad y facilidad de } \\
\text { modelado }\end{array}$ & $\begin{array}{l}\text { Es la velocidad con la que puede adaptarse a un nuevo requisito. Grado en que se puede cambiar } \\
\text { un bloque o conjunto de estos basándose en un sistema preexistente o una restricción inalterable. }\end{array}$ \\
\hline P15 & $\begin{array}{l}\text { Coherencia, consistencia y } \\
\text { acoplamiento }\end{array}$ & $\begin{array}{l}\text { Es el grado en que cada elemento hace una tarea y la hace bien. Singularidad de propósito de los } \\
\text { elementos del modelo y a la utilización de métodos y técnicas que describen las especificaciones } \\
\text { conduciendo a representaciones uniformes. }\end{array}$ \\
\hline P16 & $\begin{array}{l}\text { Extensibilidad y } \\
\text { escalabilidad }\end{array}$ & $\begin{array}{l}\text { Describe el grado en que los módulos y componentes de una arquitectura interactúan con otros } \\
\text { módulos y componentes dentro y fuera de la misma. Se describe el grado en la arquitectura pueda } \\
\text { ampliarse añadiendo variaciones. }\end{array}$ \\
\hline
\end{tabular}




\begin{tabular}{|c|c|c|}
\hline P17 & Flexibilidad & $\begin{array}{l}\text { Describe el esfuerzo necesario para modificar un artefacto o parte de la arquitectura como repuesta } \\
\text { a cambios del entorno o de la misma arquitectura. }\end{array}$ \\
\hline P18 & Mantenibilidad & $\begin{array}{l}\text { Es la facilidad con que un artefacto se puede corregir si se encuentra un error, al adaptarse a } \\
\text { cambios en el entorno o por un cambio en los requisitos. }\end{array}$ \\
\hline P19 & Visualización & $\begin{array}{l}\text { Se tiene en cuenta si el marco tiene una herramienta gráfica y si representa de forma clara los } \\
\text { componentes o vistas de la arquitectura y la interrelación entre estos. }\end{array}$ \\
\hline \multicolumn{3}{|c|}{ Dimensión No 3: Criterios de adquisición, uso, aplicación y resultados } \\
\hline $\mathrm{P} 20$ & $\begin{array}{l}\text { Tiempo potencial de } \\
\text { aplicación y evaluación }\end{array}$ & $\begin{array}{l}\text { Se tiene en cuenta el tiempo necesario para que se obtengan los primeros resultados de la aplicación } \\
\text { de la arquitectura. }\end{array}$ \\
\hline $\mathrm{P} 21$ & $\begin{array}{l}\text { Beneficios percibidos de su } \\
\text { aplicación }\end{array}$ & $\begin{array}{l}\text { En base a las necesidades y expectativas de la organización, se evalúan los beneficios que se pueden } \\
\text { obtener con la aplicación del marco de trabajo. }\end{array}$ \\
\hline $\mathrm{P} 22$ & $\begin{array}{c}\text { Relación entre ventajas y } \\
\text { desventajas }\end{array}$ & $\begin{array}{l}\text { Tiene en cuenta la relación entre las ventajas del marco de trabajo y los elementos negativos de su } \\
\text { utilización. }\end{array}$ \\
\hline
\end{tabular}

\subsection{Evaluación de los marcos de trabajo}

Resultado del filtro 1: Se reduce el listado hasta 14 marcos de trabajo para un $8,75 \%$ del total de marcos identificados. La mayor carencia de los marcos de trabajo rechazados es la no existencia de un meta modelo con solo el $12,5 \%$ de respuestas positivas.

Tabla 6 Resultados de la aplicación del filtro 2 .

\begin{tabular}{|c|c|c|c|c|}
\hline Marco & $\begin{array}{l}\text { E s t r u c t u r a l e s, } \\
\begin{array}{c}\text { conceptuales y deseno } \\
\text { diseño }\end{array}\end{array}$ & Manejo y operación & $\begin{array}{l}\text { Adquisición, uso, } \\
\text { aplicación y resultados }\end{array}$ & Puntuación total \\
\hline TOGAF & 36 & 21 & 8 & 65 \\
\hline ZIFA & 30 & 21 & 7 & 58 \\
\hline eTOM & 29 & 19 & 8 & 56 \\
\hline ITIL & 28 & 17 & 7 & 52 \\
\hline PMBOK & 31 & 13 & 8 & 52 \\
\hline FEAF & 27 & 16 & 7 & 50 \\
\hline COBIT & 26 & 13 & 7 & 46 \\
\hline SCOR & 24 & 14 & 7 & 45 \\
\hline DODAF & 23 & 11 & 7 & 41 \\
\hline $\mathrm{E} 2 \mathrm{AF}$ & 17 & 13 & 3 & 33 \\
\hline GEA & 19 & 10 & 3 & 32 \\
\hline GEAF & 14 & 13 & 5 & 32 \\
\hline MODAF & 14 & 8 & 7 & 29 \\
\hline TRAK & 15 & 6 & 7 & 28 \\
\hline
\end{tabular}

Resultado del filtro 2: Los resultados se muestran en la tabla 6 ordenados de forma descendente de acuerdo a la magnitud de sus puntuaciones. 
Analizando el patrón de la evaluación de la tabla 6 se obtienen las siguientes precisiones:

Presencia de los marcos de trabajo genéricos tradicionales: Entre los 10 marcos de trabajo de más alta puntuación se encuentran los más referenciados por la literatura. Como era de esperar los marcos Zachman y TOGAF, dos de los modelos más importantes, se encuentran encabezando el listado. Otros modelos como FEAF y DODAF se encuentran también puntuaciones por encima de los 40 puntos.

\section{Presencia de marcos de trabajo de sectores específicos:} Aunque los marcos de trabajo genéricos tradicionalmente han recibido más atención, entre los mejores evaluados en la tabla 6 resaltan marcos como e TOM, ITIL, COBIT y PMBOK que pertenecen a sectores específicos. Estos que obtienen puntuaciones altas y se colocan entre los 5 primeros.

Existencia de marcos poco conocidos: Existen marcos como E2AF, GEA y SCOR que aunque no ocupan los primeros lugares en la tabla presentan puntaciones por encima de los 30 puntos. Muchos de estos marcos son menos conocidos pero tienen potencialidades en algunas áreas según la evaluación realizada.

En la tabla 7 se muestra la puntuación alcanzada por cada marco en la dimensión de criterios estructurales, conceptuales y de diseño.

Tabla 7 Resultados de la evaluación de los criterios estructurales, conceptuales y de diseño.

\begin{tabular}{|c|c|c|c|c|c|c|c|c|c|c|c|c|}
\hline 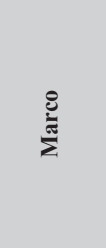 & 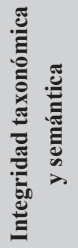 & 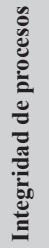 & 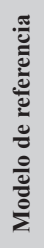 & 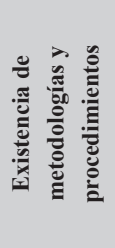 & 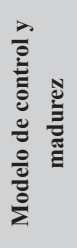 & 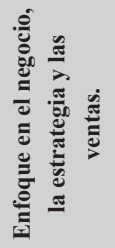 & 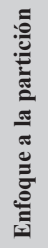 & 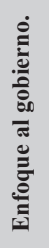 & 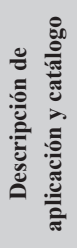 & 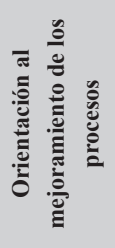 & 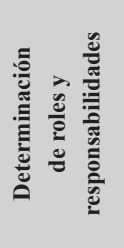 & 先 \\
\hline eTOM & 4 & 4 & 4 & 4 & 1 & 4 & 3 & 3 & 4 & 4 & 1 & 36 \\
\hline TOGAF & 4 & 4 & 4 & 1 & 1 & 3 & 2 & 1 & 4 & 3 & 3 & 30 \\
\hline ITIL & 3 & 4 & 3 & 2 & 1 & 3 & 3 & 3 & 1 & 4 & 4 & 31 \\
\hline DoDAF & 4 & 4 & 4 & 1 & 1 & 4 & 3 & 1 & 3 & 3 & 1 & 29 \\
\hline ZIFA & 3 & 4 & 1 & 1 & 1 & 3 & 3 & 4 & 1 & 4 & 3 & 28 \\
\hline COBIT & 4 & 4 & 3 & 1 & 1 & 1 & 3 & 4 & 3 & 2 & 1 & 27 \\
\hline PMBOK & 1 & 2 & 2 & 1 & 1 & 4 & 3 & 4 & 1 & 4 & 3 & 26 \\
\hline FEAF & 4 & 4 & 3 & 1 & 1 & 1 & 3 & 1 & 1 & 4 & 1 & 24 \\
\hline GEA & 4 & 4 & 3 & 1 & 1 & 1 & 3 & 1 & 1 & 1 & 3 & 23 \\
\hline SCOR & 1 & 2 & 1 & 1 & 1 & 3 & 1 & 4 & 1 & 2 & 2 & 19 \\
\hline $\mathrm{E} 2 \mathrm{AF}$ & 3 & 1 & 3 & 1 & 1 & 1 & 3 & 1 & 1 & 1 & 1 & 17 \\
\hline TRAK & 3 & 3 & 1 & 1 & 1 & 1 & 1 & 1 & 1 & 1 & 1 & 15 \\
\hline GEAF & 1 & 1 & 1 & 1 & 1 & 1 & 1 & 1 & 3 & 2 & 1 & 14 \\
\hline MODAF & 2 & 2 & 2 & 1 & 1 & 1 & 1 & 1 & 1 & 1 & 1 & 14 \\
\hline
\end{tabular}


Los 14 marcos evaluados presentan una alta integridad taxonómica y de procesos. Son marcos maduros con un desarrollo semántico evidente. La carencia de un modelo de madurez es común a todos los marcos. Con la excepción de TOGAF existe también una carencia generalizada de un procedimiento o metodología para desarrollar la arquitectura.

En la tabla 8 se muestran los resultados de la evaluación de los criterios de la dimensión manejo y operación.
Tabla 8 Resultados de la evaluación de criterios de manejo y operación

\begin{tabular}{|c|c|c|c|c|c|c|c|}
\hline 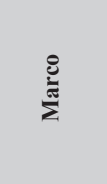 & 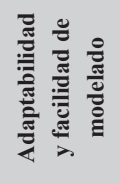 & 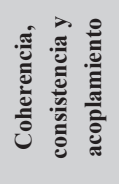 & 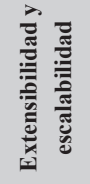 & 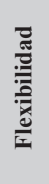 & 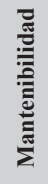 & 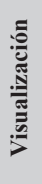 & 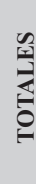 \\
\hline TOGAF & 3 & 3 & 3 & 4 & 4 & 4 & 21 \\
\hline еТОМ & 3 & 3 & 4 & 4 & 4 & 3 & 21 \\
\hline DoDAF & 1 & 3 & 3 & 4 & 4 & 4 & 19 \\
\hline ZIFA & 1 & 3 & 3 & 3 & 4 & 3 & 17 \\
\hline COBIT & 1 & 3 & 3 & 3 & 2 & 4 & 16 \\
\hline FEAF & 1 & 3 & 1 & 1 & 4 & 4 & 14 \\
\hline ITIL & 1 & 3 & 1 & 1 & 4 & 3 & 13 \\
\hline PMBOK & 1 & 3 & 1 & 1 & 4 & 3 & 13 \\
\hline E2AF & 1 & 3 & 1 & 1 & 4 & 3 & 13 \\
\hline GEAF & 1 & 3 & 3 & 1 & 3 & 2 & 13 \\
\hline GEA & 1 & 3 & 1 & 1 & 2 & 3 & 11 \\
\hline SCOR & 1 & 2 & 1 & 1 & 4 & 1 & 10 \\
\hline MODAF & 1 & 3 & 1 & 1 & 1 & 1 & 8 \\
\hline TRAK & 1 & 1 & 1 & 1 & 1 & 1 & 6 \\
\hline
\end{tabular}

Los marcos de trabajo evaluados presentan una buena mantenibilidad, un buen acoplamiento y una excelente visualización. Esta última característica está determinada por la existencia en los 14 marcos de modelos gráficos de alto perfil, tanto bidimensionales como tridimensionales. Uno de las debilidades es la dificultad para el modelado de procesos, la poca flexibilidad y la baja adaptabilidad. Excepto TOGAF y ZACHMAN que son flexibles en cierto grado, los demás marcos presentan el inconveniente de la complejidad semántica y estructural. También se presenta la rigidez de algunos de sus componentes o vistas.

En la tabla 9 se muestran los resultados de la evaluación de los criterios de la dimensión adquisición, uso, aplicación y resultados. 
Tabla 9 Resultados de la evaluación de criterios de adquisición, uso, aplicación y resultados

\begin{tabular}{|c|c|c|c|c|}
\hline Marco & $\begin{array}{l}\text { Tiempo potencial de } \\
\text { aplicación y evaluación }\end{array}$ & $\begin{array}{l}\text { Beneficios percibidos } \\
\text { de su aplicación }\end{array}$ & $\begin{array}{c}\text { Relación entre ventajas } \\
\text { y desventajas }\end{array}$ & TOTALES \\
\hline етОм & 4 & 1 & 3 & 8 \\
\hline ITIL & 4 & 1 & 3 & 8 \\
\hline DoDAF & 4 & 1 & 3 & 8 \\
\hline TOGAF & 3 & 1 & 3 & 7 \\
\hline ZIFA & 3 & 1 & 3 & 7 \\
\hline PMBOK & 3 & 1 & 3 & 7 \\
\hline FEAF & 3 & 1 & 3 & 7 \\
\hline COBIT & 3 & 1 & 3 & 7 \\
\hline GEA & 3 & 1 & 3 & 7 \\
\hline MODAF & 3 & 1 & 3 & 7 \\
\hline TRAK & 3 & 1 & 3 & 7 \\
\hline GEAF & 1 & 1 & 3 & 5 \\
\hline SCOR & 1 & 1 & 1 & 3 \\
\hline $\mathrm{E} 2 \mathrm{AF}$ & 1 & 1 & 1 & 3 \\
\hline
\end{tabular}

De forma general el tiempo de aplicación y desarrollo de los marcos es evaluado positivamente. Existen marcos como el eTOM y DODAF que presentan un tiempo de aplicación más extendido. También se evalúa positivamente la relación ventajas y desventajas. Todos los marcos del listado presentan más aspectos positivos que negativos. El criterio que más pobremente se desempeña es el relacionado con los beneficios percibidos de su aplicación. Sin lugar a dudas los marcos de trabajo aportan a las organizaciones donde son aplicados pero este impacto aún no ha sido adecuadamente demostrado.

\section{Valoración del aporte de la investigación}

Los aportes de la investigación se enfocan tanto en el mundo académico como empresarial. El trabajo contribuye en las siguientes dimensiones: Manejo de la diversidad, orientación metodológica sobre análisis y/o selección y actualización sobre el contexto actual de la arquitectura empresarial.

Manejo de la diversidad: Como se explica en el epígrafe 2 la pluralidad y variedad es una de las principales dificultades que presentan los marcos de trabajo de arquitectura empresarial para su aplicación práctica. Excepto (Sultanow et al., 2016), que plantea un listado de 55 marcos de trabajo, el resto de los trabajos consultados incluyen en sus estudios pequeños grupos de modelos. Muchos estudios repiten sobre un grupo representativo de marcos de trabajo como son TOGAF, Zachman, FEAF, DODAF, entre otros, lo que provoca críticas en cuanto a la sobrevaloración de esos modelos sobre otros (Kotusev, 2018a) (Kotusev, 2019b). El listado de marcos de trabajo incluido en este estudio se ofrece un panorama amplio que representa la verdadera diversidad, más allá de los modelos de moda o los más divulgados. Se pone a disposición del usuario de la arquitectura empresarial no solo el listado actualizado de marcos de trabajo, sino que se ofrece información sobre estos adecuadamente indexada y catalogada. Esto contribuye al ahorro de tiempo y recursos. La información sobre los marcos de trabajo se encuentra muy dispersa y aquí se brinda de forma integrada. Con los resultados de esta investigación en sus manos, los arquitectos empresariales pueden confrontar muchos marcos de trabajo en sus vistas, procesos, elementos y metodologías.

Orientación metodológica: La investigación aporta un método de evaluación de marcos de trabajo que incluyendo criterios de métodos ya establecidos resuelve varias de sus 
incongruencias. Este incluye descripciones teóricas de cada criterio que permite establecer claramente las diferencias entre los marcos de trabajo evaluados. Abarca criterios no solo funcionales sino que los integra con criterios de explotación y uso realizando una evaluación más integrada. La propuesta metodológica establece pautas claras sobre que es un marco de trabajo adecuado. Ofrece un método estructurado fácil de utilizar en la práctica integrando buenas prácticas y criterios validados por varios trabajos e investigaciones.

Actualización sobre el contexto actual: Aunque no necesariamente la aplicación de la arquitectura empresarial se relaciona con la utilización de los marcos de trabajo, no cabe dudas de que estos están presentes en la mayoría de sus iniciativas. Los estudiosos de la arquitectura empresarial tienen en esta investigación una base documental actualizada para hacer diversos análisis como la mutabilidad de los modelos, las derivaciones existentes, la comparación de meta-modelos, etc.

\section{Conclusiones}

La arquitectura empresarial ha aportado nuevos matices a las ciencias empresariales. Sus contribuciones para el manejo de complejidad de las organizaciones, la integración de procesos con las tecnologías, el aumento de las capacidades estratégicas y su apoyo a la transformación digital son un hecho. A pesar de esto, constituye un reto para la arquitectura empresarial hacer tangibles los aportes en la práctica organizacional y los marcos de trabajo son imprescindibles en este sentido. Estos han sido fundamentales en el auge y desarrollo de arquitectura empresarial. No solo constituyen representaciones de arquitecturas tipo, se han convertido en modelos de buenas prácticas que establecen pautas de cómo deben ser las arquitecturas de éxito.

Dada la complejidad del mundo de los marcos de trabajo por su variedad y confusión, establecer pautas claras sobre cuáles son los modelos que están disponibles y aportar información actualizada sobre los mismos tiene un gran valor práctico. El listado general actualizado de marcos de trabajo con su gran cúmulo de información resultante, la metodología propuesta para evaluar los marcos y el análisis de la evaluación constituyen un paquete útil para cualquier profesional que se acerca a la arquitectura empresarial. Una de las causas más comunes de retraso o fracaso de programas de arquitectura empresarial es la imposibilidad de manejar de forma coherente tanta diversidad de arquitecturas, marcos y metodologías. Con los resultados de esta investigación se contribuye a atenuar los efectos de esta barrera y potenciar lo que todo profesional espera de la arquitectura empresarial: la mejora real en la práctica de las organizaciones.

\section{Bibliografía}

1. Abdallah, A., \& Abran, A. (2019). «Enterprise architecture measurement: An extended systematic mapping Study». I.J. Information Technology and Computer Science, 9, pp 9-19.

2. Abdallah, S., \& Galal-Edeen, G. H. (2009). Towards a framework for enterprise architecture frameworks comparison and selection. University in Egypt.

3. Abdullah, S. (2017). «A proposed model to measure the impact of business architecture». Cogent Business \& Management, 4, pp 1-8.

4. Abunadi, I. (2019). «Enterprise architecture best practices in large corporations». Information, 10 (293), pp 1-26.

5. Aier, S., Fischer, C., \& Winter, R. (2011). Construction and evaluation of a meta-model for enterprise architecture design principles. International Conference on Wirtschaftsinformatik, Zurich, Switzerland.

6. Aier, S., \& Schelp, J. (2010). A reassessment of enterprise architecture implementation. Germany: Springer Verlag Heidelberg.

7. Ajer, A. K. S. (2020). Institutional perspective on introducing enterprise architecture. The case of the norwegian hospital sector [tesis de doctorado]. University of Agder.

8. AlDhaheri, S. (2011). A study on enterprise architecture framework effects on business processes automation [tesis de maestría]. The British University of Dubai. Faculty of Engineering \& IT.

9. Almisned, F. A. (2017). Extending the capacity of enterprise architecture management frameworks: towards a model-driven handling of dynamics [tesis de doctorado]. Department of Informatics in the Faculty of Natural \& Mathematical Sciences. King's College London. https://kclpure.kcl.ac.uk/portal/

10. Alwadain, A., Fielt, E., Korthaus, A., \& Rosemann, M. (2014). «A critical realist perspective of enterprise architecture evolution: conditioning and outcomes». Australasian Journal of Information Systems, 18(3), pp 213-226.

11. Armour, F., \& Kaisler, S. (2017). Enterprise architecture and business process analysis. Hawaii International Conference on System Sciences, United States of America. http://hdl.handle.net/10125/41746ISBN 
12. Azizi, L., \& Sumitra, I. D. (2019). Designing of enterprise architecture for interior furniture production based on TOGAF 9.1. IOP Conference Series: Materials Science and Engineering

13. Baharudin Jusuf, M., \& Kurnia, S. (2017). Understanding the benefits and success factors of enterprise architecture. Hawaii International Conference on System Sciences, United States of America, pp 4887-4896. http://hdl. handle.net/10125/41755ISBN:

14. Banaeianjahromi, N. (2018). On the role of enterprise architecture in enterprise integration [tesis de doctorado]. Lappeenranta University of Technology.

15. Banaeianjahromi, N., \& Smolander, K. (2017). Lack of communication and collaboration in enterprise architecture development. United States of America: Springer Science+Business Media. https://doi. org/10.1007/s10796-017-9779-6

16. Barekat, V., Nejad, E. B., \& Alavi, S. E. (2013). «Definition of zachman framework cells based on service oriented architecture». International Journal of Scientific and Research Publications, 3(9), pp 1-8.

17. Bernard, S. A. (2012). An introduction to enterprise architecture (Third Edition). United States of America: Author House.

18. Bernus, P., \& Doumeingts, G. (2010). Enterprise architecture, integration and interoperability. World Computer Congress, Australia.

19. Bernus, P., \& Noran, O. (2010). A metamodel for enterprise architecture. HAL. Archives ouvertes. https:// hal.inria.fr/hal-01054819

20. Bui, Q. N. (2017). «Evaluating Enterprise architecture frameworks using essential elements». Communications of the Association for Information Systems, 41(6.), pp 121-149.

21. Cabrera, A., Abad, M., Jaramillo, D., Gomez, J., \& Carrillo, J. é. (2016). «Definition and implementation of the enterprise business layer through a business reference model, using the architecture development method ADM-TOGAF». Trends and Applications in Software Engineering, 405, pp 111-121.

22. Carr, D. (2018, mayo). «State of Enterprise Architecture Survey: Results and Findings». Enterprise Architecture Professional Journal (Special Edition).

23. Caruso, M. J. (2019). Strategies to improve adoption of the federal enterprise architecture framework [tesis de doctorado]. Walden University.
24. Chakraborty, P., \& Sarkar, A. (2017). Context driven approach for enterprise architecture framework. United States of America: Springer International Publishing AG.

25. Chorafas, D. N. (2002). Enterprise architecture and new generation information systems. United States of America: CRC Press LLC.

26. Cretu, L. G. (2014). Designing enterprise architecture frameworks. integrating business processes with IT Infrastructure. Unites States of America: (Taylor \& Francis), Apple Academic Press, Inc.

27. Dube, M. R., \& Dixit, S. K. (2011, agosto). «Comprehensive measurement framework for enterprise architectures». International Journal of Computer Science \& Information Technology (IJCSIT), 3(4), pp 71-92.

28. Erdenebold, T., Rho, J. J., \& Hwang, Y. M. (2019). «Blockchain reference model and use case for supply chains within enterprise architecture». Journal of Information Technology and Architecture, 16(1), pp $1-10$.

29. Essien, J. (2019). «Goal-oriented model driven enterprise architecture validation approach». International Journal of Advanced Research and Publications, pp 107-114.

30. Farazmand, E., \& Moeini, A. (2011). A framework for knowledge management architecture. International Conference on Enterprise Information Systems, Iran, pp 425-430. https://doi.org/10.5220/0003492504250430

31. Ferreira de Santana, A. T. (2017). Enterprise architecture analysis based on network paradigm: a framework proposal and empirical evaluation [tesis de doctorado]. Universidade Federal de Pernambuco.

32. Fischer, C., Winter, R., \& Aier, S. (2010). What is an enterprise architecture principle? towards a consolidated definition. Computer and Information Science, St. Gallen, Switzerland, pp 193-205.

33. Foorthuis, R. (2012). Project compliance with enterprise Architecture [tesis de doctorado]. Utrecht University, Utrecht, Netherlands. Department of Information and Computing Sciences, Organization and Information.

34. Fratila, L.-A. (2020). «Enterprise architecture and corporate governance a cohesive approach towards cloud migration in the banking industry». International Journal of Economics, Commerce and Management, VIII (5). http://ijecm.co.uk/ 
35. Gampfer, F. (2019). Investigation on the future of enterprise architecture in dynamic environments [tesis de doctorado]. Universidad de Murcia.

36. Giachetti, R. E. (2015). «Evaluation of the DoDAF metamodel's support of systems engineering». Procedia Computer Science, 4, pp 254-60.

37. Gokhale, A. (2010). Increasing effectiveness of the zachman framework using the balanced scorecard [tesis de Maestría]. Purdue University.

38. Halawi, L., McCarthy, R., \& Farah, J. (2018). Where we are with enterprise architecture. Conference on Information Systems Applied Research, United States of America.

39. Hameed, K., Shah, H., Ahsan, K., \& Yang, W. (2010). «An enterprise architecture framework for mobile Commerce». IJCSI International Journal of Computer Science Issues, 7(5), pp 6-12.

40. Harrison, R. (2015). TOGAF v9. Certified. Study guide 3rd Edition (The Open Group). United States of America: Van Haren Publishing. www.opengroup.org

41. Hylving, L., \& Bygstad, B. (2018). Responding to enterprise architecture initiatives: loyalty, voice and exit. Hawaii International Conference on System Sciences, pp 2363-2372.

42. Jayakrishnan, M., Mohamad, A. K., \& Abdullah, A. (2019). «Enterprise architecture embrace digital technology in malaysian transportation industry». International Journal of Engineering and Advanced Technology, 8(4), pp 852-859.

43. Joubert, F. (2010). Knowledge assets in enterprise architecture [tesis de maestría]. University of Stellenbosch. Faculty of Arts and Social Sciences Department of Information Science.

44. Kamoun, F. (2013). «Rethinking the role of enterprise architecture during times of economic downturn: a dynamic capabilities approach». Journal of Information Technology Management, XXIV (1), pp 26-48.

45. Kar, S., \& Thakurta, R. (2018). Planning for digital transformation: implications for institutional enterprise architecture. Twenty-Second Pacific Asia Conference on Information Systems, Japan. https://aisel.aisnet.org/ pacis 2018

46. Kimpimäki, H. (2014). Enterprise architecture in practice: from it concept towards enterprise architecture leadership [Tesis de doctorado]. Tampere University of Technology.
47. Kotusev, S. (2016). Enterprise Architecture Frameworks: The fad of the century. British Computer Society http:// www.bcs.org/content/conWebDoc/56347

48. Kotusev, S. (2017). «Enterprise Architecture: What Did We Study? » International Journal of Cooperative Information Systems, 26(4), pp 1-84.

49. Kotusev, S. (2018a). TOGAF: Just the next fad that turned into a new religion. Pragmatic EA Ltd.

50. Kotusev, S. (2018b). «TOGAF-based enterprise architecture Practice: An exploratory case study». Communications of the Association for Information Systems, 43(20). https://aisel.aisnet.org

51. Kotusev, S. (2018c). «Enterprise Architecture: A reconceptualization is needed». Pacific Asia Journal of the Association for Information Systems, 10(4), pp 1-36.

52. Kotusev, S. (2019a). «Enterprise architecture and enterprise architecture artifacts: Questioning the old concept in light of new findings». Journal of Information Technology, 34(2), pp 102-128.

53. Kotusev, S. (2019b). «Fake and Real Tools for Enterprise Architecture: The Zachman Framework and business capability model». Enterprise Architecture Professional Journal http://www.bcs.org/content/conWebDoc/59399

54. Kotusev, S., Singh, M., \& Storey, I. (2015). Consolidating enterprise architecture management research. Hawaii International Conference on System Sciences, United States of America, pp 4069-4078. https://doi.org/10.1109/HICSS.2015.489

55. Lange, M. hias, Mendling, J., \& Recker, R. (2012). Realizing benefits from enterprise architecture: a measurement model. http://aisel.aisnet.org/ecis2012

56. Lankhorst, M. (2009). Enterprise architecture at work. modelling, communication and analysis (Second Edition). Berlin: Springer-Verlag Berlin Heidelberg.

57. Laschitza, J., \& Undén, M. (2017). Enterprise architecture implementation [Tesis de maestría]. University of Gothenburg.

58. Lauvrak, S., Michaelsen, V. M., \& Olsen, D. H. (2017). «Benefits and Challenges in enterprise architecture management: A case study of the Norwegian labour and welfare administration». Bibsys Open Journal Systems, 25(1).

59. Lê, L. (2020). «Diagramming multi level service oriented enterprise architecture». Computer Science, 1(14), pp 1-25. 
60. Llanos Cuenca, A. B., \& Ortiz, A. (2011). «An enterprise engineering approach for the alignment of business and information technology strategy». International Journal of Computer Integrated Manufacturing. 24(11), pp 974992.

61. Londoño Salazar, J. E. (2014). Modelo funcional de integración de la arquitectura empresarial de ' $n$ ' entidades alrededor de un grupo empresarial. un enfoque de orientación a servicios y modelado de capacidades de negocio [tesis de doctorado]. Universidad Nacional de Colombia.

62. Lukhele, C. F., Ngassam, E. K., \& Osunmakinde, I. (2015).A framework for measuring the value of enterprise architecture in South African telecommunications organizations. AFRICOMM, South Africa, pp 282-292.

63. Mahmood, Z., \& Hill, R. (2011). Cloud computing for enterprise architectures. United Kingdom: Springer Verlag London Limited.

64. Marini, G. (2019b). Enterprise architecture and digital transformation [tesis de maestría]. NOVA, School of Business and Economics.

65. Masuda, Y., \& Viswanathan, M. (2019). Enterprise architecture for global companies in a digital it era. Adaptive integrated digital architecture framework (AIDAF). Springer Nature Singapore. https://doi. org/10.1007/978-981-13-1083-6

66. McDowall, J. D. (2019). Complex enterprise architecture. A new adaptive systems approach (Susan McDermott). Apress Media LLC. https://doi.org/10.1007/978-14842-4306-0

67. Mendieta Matute, M. I. (2014). Propuesta de framework de arquitectura empresarial para pymes basado en un análisis comparativo de los frameworks Zachman y TOGAF [tesis de maestría]. Universidad de Cuenca.

68. Minoli, D. (2008). Enterprise Architecture A to Z. Frameworks, business process modeling, soa, and infrastructure technology. United States of America: Auerbach Publications. Taylor \& Francis Group, LLC.

69. Mokone, C. B., Eyitayo, O. T., \& Masizana, A. (2019). «Decision support process for selection of an optimal enterprise architecture framework for e-government implementation». Journal of E-Government Studies and Best Practices, 19, pp 1-4.
70. Mondorf, A., \& Wimmer, M. A. (2017). Contextual Components of an enterprise architecture framework for pan-european e-government services. Hawaii International Conference on System Sciences, United States of America, pp 2933-2942. http://hdl.handle. net/10125

71. Moscoso-Zea, O., \& Lujan-Mora, S. (2017). «Metodologías sugeridas de evaluación y selección de software de arquitectura empresarial para la digitalización del conocimiento». Enfoque UTE, 7(1), pp 315-328.

72. Nardello, M. (2019). Enterprise architecture for digital manufacturing EA models and an automated EA modelling method to support industry 4.0 transformation. Aalborg Universitet.

73. Niemann, K. D. (2005). From enterprise architecture to IT governance. Germany: Friedr. Vieweg \& Sohn Verlag.

74. Niemi, E. (2006). Enterprise architecture benefits: Perceptions from literature and practice. IBIMA Conference Internet \& Information Systems in the Digital Age, Italy.

75. Niemi, E. (2016). Enterprise architecture benefit realization. [tesis de doctorado]. Tampere University of Technology.

76. Niemi, E., \& Pekkola, S. (2019). The benefits of enterprise architecture in organizational transformation. https://doi.org/10.1007/s12599-019-00605-3

77. Nilsen, O. R. (2012). Enterprise content management: an analysis of contemporary practice and its relationships with enterprise architecture [tesis de maestría]. University of Agder.

78. Noran, O. (2018). «An analysis of the Zachman Framework for enterprise Architecture from the GERAM perspective». IFAC Annual Reviews in Control, 27(2), pp 163-183.

79. Nygård, M., \& Olsen, D. H. (2016). «Enterprise architecture implementation challenges: an exploratory study of the Norwegian health sector». Bibsys Open Journal Systems, 24(1), pp 28-30.

80. Okhrimenko, A. (2017). Comparing enterprise architecture frameworks. a case study at the estonian rescue board [tesis de maestría]. University of Tartu. 
81. Oliveira Barbosa, A., Santana, A., Hacks, S., \& von Stein, N. (2019). A taxonomy for enterprise architecture analysis research. 21st International Conference on Enterprise Information Systems, Unites States of America, pp 493-504. http:/urn.kb.se/ resolve?urn=urn:nbn:se:kth:diva-255629

82. Orabona, A. E. (2016). Building a foundation for the implementation of an enterprise architecture for the Argentinian Army [tesis de maestría]. Naval Postgraduate School.

83. Pähler, P., Morency, M. C., \& Lapalme, J. (2019). «Examination of explicit definitions of enterprise architecture». International Journal of Engineering Business Management, 11, pp 1-18.

84. Pasaribu, F. A., Sipahutar, J. H., \& Situmorang, B. P. (2019). Designing enterprise architecture in hospitals group.

85. Perroud, T., \& Inversini, R. (2013). Enterprise architecture patterns. practical solutions for recurring IT-architecture problems. Germany: Springer-Verlag Berlin Heidelberg.

86. Prayitno, O. T. (2019). «Planning of Higher Education Information Technology Strategy Using TOGAF (A Case Study at AMN Cilacap) ». Indonesian Journal of Information Systems (IJIS), 2(1), pp 67-79.

87. Priyanto, A. A. (2013). Bringing Bringing Enterprise Architecture to the Boardroom [tesis de maestría]. University of Twente.

88. Proper, E., Lankhorst, M. M., Schönherr, M., Barjis, J., \& Overbeek, S. (2012). Trends in Enterprise Architecture Research. Springer-Verlag.

89. Qazi, H., Javed, Z., Majid, S., \& Mahmood, W. (2019). "A detailed examination of the enterprise architecture frameworks being implemented in Pakistan». I.J. Modern Education and Computer Science, 19 (9), pp 44-53.

90. Qurratuaini, H. (2017). Designing enterprise architecture based on TOGAF 9.1 framework. IOP Conference Series: Materials Science and Engineering. International Conference on Engineering and Applied Technology (ICEAT), United States of America.

91. Randall, N. (2018). Enterprise architecture content strategy.
92. Rehring, K., \& Ahlemann, F. (2020). Evaluating the user experience of an augmented reality prototype for enterprise architecture. International Conference on Wirtschaftsinformatik, Potsdam, Germany. https://doi. org/10.30844/wi_2020_i3-rehring

93. Roach, T. (2011). CAPSICUM. A semantic framework for strategically aligned business architecture. UNSW. School of Information Systems and Technology Management.

94. Rogier, P. F., van de Wetering, R., \& Joosten, S. (2020). Enterprise architecture alignment.

95. Rohloff, M. (2011). Integrating innovation into enterprise architecture Management. International Conference on Wirtschaftsinformatik, Zurich, Switzerland.

96. Ross, J. W., Weill, P., \& Robertson, D. C. (2006). Enterprise architecture as strategy. creating a foundation for business execution. United States of America: Harvard Business School Press.

97. Ross, J. W., Weill, P., \& Robertson, D. C. (2014). Enterprise architecture as strategy.

98. Rouhani, B. D., \& Nikpay, F. (2012). «Agent-oriented enterprise architecture: new approach for enterprise architecture». International Journal of Computer Science Issues, 9(6), pp 331-334.

99. Saint-Louis, P. (2019). Investigation of the lack of common understanding in the discipline of enterprise architecture [tesis de doctorado]. École de Technologie Supérieure Université du Québec.

100.Saint-Louis, P., \& Lapalme, J. (2018). «An exploration of the many ways to approach the discipline of enterprise architecture». International Journal of Engineering Business Management, 10, pp 1-26.

101.Sanchez Puchol, F., \& Pastor Collado, J. A. (2017). A first literature review on enterprise reference architecture. The Mediterranean Conference on Information Systems (MCIS), Genoa, Italy. http://aisel. aisnet.org/mcis2017/15

102.Sandoval, F., Galvez, V., \& Moscoso, O. (2017). «Desarrollo de arquitectura empresarial usando un framework con enfoque ágil». Enfoque UTE, 1, pp 135147. 
103.Sari, T. R., Rahmawati, E., \& Harafani, H. (2019). «TOGAF ADM to improve the promotion of farm edutourism in Pondok Rangon area». Journal Publications \& Informatics Engineering Research, 3(2).

104.Schekkerman, J. (2006). Enterprise architecture assessment guide. Version 2.2. Institute for Enterprise Architecture Development.

105.Schekkerman, J. (2011). Enterprise architecture tool selection guide. Institute For Enterprise Architecture Developments (IFEAD).

106. Schekkerman, J. (2004). How to survive in the jungle of enterprise architecture frameworks: creating or choosing an enterprise architecture framework (Second Edition). United States of America: Trafford.

107.Scherer, S., \& Wimmer, M. A. (2017). «E-participation and enterprise architecture frameworks: An analysis». Information Polity, pp 147-161.

108.Selcan, V., \& Buchalcevova, A. (2013). An analysis of enterprise architecture frameworks from a coherent enterprise description viewpoint. International Conference on Information Systems Development. Prague, Czech Republic.

109.Sessions, R. (2008). Simple architectures for complex enterprises. United States of America: Microsoft Press.

110.Sessions, R. (2011). Revamping public sector it procurement to favour success and small business. consortium for untangling enterprise complexity.

111.Sessions, R., \& deVadoss, J. (2014). A comparison of the top four enterprise architecture approaches in 2014.

112.Sidorova, A., \& Kappelman, L. (2010). Realizing the benefits of enterprise architecture: An Actor-Network Theory Perspective. College of Business, University of North Texas.

113.Simon, D., Fischbach, K., \& Schoder, D. (2013). An exploration of enterprise architecture research. Communications of the Association for Information Systems, 32.

114.Sofyana, L., \& Putera, A. (2019). Business architecture planning with TOGAF framework. IOP Publishing.

115.Stroud, R. O. (2019). Complexity frameworks in enterprise design [Tesis de doctorado]. Texas Tech University.
116.Sukur, A. (2020). Enterprise architecture routinization and use of enterprise architecture standards to achieve information technology flexibility and enterprise agility [Tesis de doctorado]. Capella University.

117.Sultanow, E., Schroeder, K., Brockmann, C., \& Cox, S. (2016). A multidimensional classification of 55 enterprise architecture frameworks. Twenty-second Americas Conference on Information Systems, San Diego. EEUU.

118.Tamm, T., Seddon, P. B., Shanks, G., \& Reynolds, P. (2011). «How does enterprise architecture add value to organisations? » AIS Journals at AIS Electronic Library (AISeL), 28(10), pp 141-168.

119.The Open Group. (2018). The TOGAF Standard, Version 9.2.

120.Open Group. (2011). TOGAF Version 9.1 A Pocket Guide.

121.Thea Nisaa', A. S., Asti, A. N. F., \& Muharman, L. (2019). IT Roadmap to improve business strategy using TOGAF ADM: A case study of government owned Electricity Company. IOP Publishing.

122.Theuerkorn, F. (2005). Lightweight enterprise architectures. United States of America: CRC Press LLC.

123.Tome, P., \& Costa, E. (2010). TREEAD A tool that enables the re-use of experience in enterprise architecture description. International Conference on Enterprise Information Systems, Artificial Intelligence and Decision Support Systems, Portugal, pp 332-343.

124.Urbaczewski, L., \& Mrdalj, S. (2018). «A comparison of enterprise Architecture Frameworks». Issues in Information Systems, VII (2), pp 18-23.

125.Vargas, A. (2015). Propuesta de arquitectura empresarial en el contexto de colaboración jerárquica para el soporte a la toma de decisiones en situaciones de eventos inesperados [tesis de doctorado]. Universitat Politècnica de València.

126.Vermeulen, R. J. M. (2013). Design of an enterprise architecture for information requirements identification: An exploratory study within the high-tech industry. TUE. School of Industrial Engineering. 
127.Werewka, J. (2018). Developing conformance between project management and enterprise architecture governance on the basis of a PMBOK case. Advances in Intelligent Systems and Computing. International Conference on Information Systems Architecture and Technology ISAT 2017, Poland.

128.Winter, K., Buckl, S., Matt hes, F., \& Schweda, C. M. (2010). Investigating the state-of-the-art in enterprise architecture management methods in literature and practice. Mediterranean Conference of Information Systems, Germany.

129.Winter, R., \& Fischer, R. (2007). Essential layers, artifacts, and dependencies of enterprise architecture. Journal of Enterprise Architecture.

130. Yoganingrum, A., Sensuse, D. I., \& Murni, A. (2013). "A taxonomy of enterprise architecture framework for indonesian SMEs». International Journal of Computer Science Issues, 10 (2).
131.Zachman, J. A. (1987). «A framework for information systems architecture». IBM Systems Journal, 26(3), pp 454-470.

132.Zachman, John A. (2003). The Zachman Framework for enterprise architecture: primer for enterprise engineering and manufacturing. OMG BRWG's.

133.Zachman, John A. (2011). The practice of enterprise architecture. Zachman International.

134.Zarvic, N., \& Wieringa, R. (2006). An integrated enterprise architecture framework for business-IT alignment. BUSITAL'06, Netherlands, pp 262-270. 